\title{
Synthesis, Nematicidal and Antifungal Properties of Hybrid Heterocyclics
}

\author{
Avula Srinivas, ${ }^{1, \star}$ Malladi Sunitha, ${ }^{1}$ Pulluri Karthik ${ }^{1}$ \\ and K. Vasumathi Reddy ${ }^{2}$ \\ ${ }^{1}$ Department of Chemistry, Vaagdevi Degree \& PG College \\ ${ }^{2}$ Department of Zoology, Vaagdevi Degree \& PG College Kishanpura, Warangal, Telangana, India 506001 \\ *Corresponding author: E-mail: avula.sathwikreddy@gmail.com
}

Received: 28-08-2017

\begin{abstract}
A new series of 5-((3aR,5S,6S,6aR)-6-((1-(4-chlorophenyl)-1H-1,2,3-triazol-4-yl)methoxy)-2,2-dimethyltetrahydrofuro[2,3-d][1,3] dioxol-5-yl)-3-(4-fluorophenyl)-2,6-diphenyl-3,3a,5,6-tetrahydro-2H-pyrazolo[3,4- $d$ thiazoles 10a-r was synthesized by the reaction of chalcone derivatives of $2-((3 \mathrm{a} R, 5 S, 6 S, 6 \mathrm{a} R)-6-((1-(4-\mathrm{chlorophenyl})-1 \mathrm{H}-1,2,3$-triazol4-yl)methoxy)-2,2-dimethyltetrahydrofuro[2,3-d][1,3]dioxol-5-yl)-3-phenylthiazolidin-4-one 9 with aryl/alkyl hydrazines. The chemical structures of newly synthesized compounds were elucidated by IR, NMR, MS and elemental analysis. The compounds 10a-r were evaluated for their nematicidal activity against Dietylenchus myceliophagus and Caenorhabditis elegans by aqueous in vitro screening technique. Among them, compounds containing $N$-benzylpyrazole moiety $(\mathbf{1 0 d}, 10 \mathbf{j}, 10 \mathbf{p})$, and $N$ - methylpyrazole moiety $(10 \mathrm{f}, 10 \mathrm{i}, 10 \mathrm{r})$ showed significant nematicidal activity against both tested nematodes with $\mathrm{LD}_{50} 160-210 \mathrm{ppm}$, almost equal to oxamyl standard. Further, these compounds 10a-r were screened for their antifungal (MZI, MIC, and MFC) activity against four fungal organisms viz, Candida albicans (ATCC 102331), Aspergillus fumigates (HIC 6094), Trichophyton rubrum (IFO 9185) and Trichophyton mentagrophytes (IFO 40996). Most of the new compounds showed appreciable activity against the tested fungi, and emerged as potential molecules for further development.
\end{abstract}

Keywords: Hybrid heterocyclics, click reaction, Knovenagel condensation, cyclisation, nematicidal activity, antifungal activity

\section{Introduction}

1,2,3-Triazoles are one of the most important classes of heterocyclic organic compounds, which are reported to be present in a plethora of biological activities in diverse therapeutic areas. ${ }^{1}$ The 1,2,3-triazole motif is associated with diverse pharmacological activities, such as antibacterial, antifungal, hypoglycemic, antihypertensive and analgesic properties. Polysubstituted five-membered aza heterocycles rank as the most potent glycosidase inhibitors. ${ }^{2}$ Further, this nucleus in combination (or linked) with various other classes of compounds, such as amino acids, steroids, aromatic compounds, carbohydrates etc., became prominent in having various pharmacological properties. $^{3}$ 1,2,3-Triazole modified carbohydrates have became easily available after the discovery of the $\mathrm{Cu}(\mathrm{I})$ catalyzed azide-alkynes 1,3-dipolar cycloaddition reaction $^{4}$ and quickly became a pronounced class of non-nat- ural sugars. The chemistry and biology of triazole modified sugars is dominated by triazole glycosides. ${ }^{5}$ Therefore, the synthesis and investigation of biological activity of 1,2,3-triazole glycosides is an important objective, which also received a considerable attention by the medicinal chemists.

Thiazoles are familiar group of heterocyclic compounds possessing a wide variety of biological activities and their utility as medicine is very much estabilished. ${ }^{6}$ Thiazole nucleus is also an integral part of all the available pencillins which have revolutionized the therapy of bacterial diseases. ${ }^{7}$ Furthurmore, there has been considerable interest in the chemistry of thiazolidine-4-one ring system which is the core structure in various synthetic pharmaceuticals displaying a broad spectrum of biological activities. $^{8-10}$ The thiazolidine-4- one ring also occurs in nature; thus actithiazic acid isolated from Streptomycis strains exhibits highly specific in vitro activity against mycobacteri- 
um Tuberculosis. ${ }^{11}$ Thiazolidine-4-ones are known to exhibit diverse bioactivities, such as antiplatelet activating factor, ${ }^{12}$ antihistaminic, ${ }^{13} \mathrm{COX}$ inhibitory, ${ }^{14} \mathrm{Ca}^{+2}$ channel blocking, ${ }^{15}$ PAF antagonist, ${ }^{16}$ cardioprotective, ${ }^{17}$ anti-ischemic ${ }^{18}$ and nematicidal activities. ${ }^{19}$ Moreover, pyrazoles and their derivatives could be considered as possible antimicrobial agents. ${ }^{20}$ The other derivatives display antidepressant, $^{21}$ antiarthritic ${ }^{22}$ and cerebroprotecting ${ }^{23}$ properties. Some aryl pyrazoles were reported to be non-nucleoside human immunodeficiency virus (HIV-1) reverse transcriptase inhibitor, ${ }^{24} \mathrm{COX}-2$ inhibitor, ${ }^{25-27}$ activator of the nitric oxide receptor and to have soluble guanylate $c y-$ clase activity. ${ }^{28}$

Nematodes are tiny worms, some of them are plant parasites, and can play an important role in the predisposition of the host plant to the invansion by secondary pathogens. ${ }^{29}$ Plants attacked by nematodes show retarded growth and development, as well as loss in the quality and quantity of the harvest. The namaticides currently still in use are slated for reduction due to the environmental problems, and human and animals health concerns. For example, effective namaticides, such as dibromochloropropane (DBCD) and ethylene dibromide (EDB) have been withdrawn from the market due to their deleterious effects on humans and the environment. Methyl bromide, the most effective and widely used fumigant for soil-borne pests including nematodes, has already been banned.

The use of nonfumigant nematicides, based on organophosphates and carbamates, is expected to increase concomitantly with the withdrawal of methyl bromide, which will bring about new environmental concerns. In fact, the highly toxic Aldicarb used to control insects and nematodes has been detected in ground water. ${ }^{30}$ Therefore, alternative nematode control methods or less toxic nematicides need to be developed. ${ }^{31}$ One way of searching for such nematicidal compounds is to screen naturally occurring compounds in plants. Several such compounds, e.g. alkaloids, sesquiterpenes, diterpenes, polyacetylenes have nematicidal activity. ${ }^{32-33}$ For example, a-terthienyl is a highly effective nematicidal compound. Other compounds with nematicidal activity have been isolated from plants, mainly from the family Asteraceae. ${ }^{32-33}$ However, compounds of plant origin and their analogs have not been developed into commercial nematicides yet; hence there is a need to develop commercial syntheses.

Following the successful introduction of antimicrobial and nematicidal agents, inspired by the biological profile of triazoles, thiazolidinones, pyrazoles and their increasing importance in pharmaceutical and biological fields and in continuation of our work on biologically active molecules ${ }^{34-48}$ and in order to enhance the biological activity of triazoles, thiazolidinones and pyrazole moieties, it was thought to be of interest to accomodate triazole, thiazolidinones and pyrazole moieties in single molecular framework. In this article we report the synthesis of a new class of hybrid heterocycles $\mathbf{1 0 a}-\mathbf{g}$ in good yields and their evaluation for in vitro antifungal and nematicidal activity.

\section{Results and Discussion}

The key intermediate $\mathbf{8}$ required for the synthesis of title compound was prepared according to the procedure outlined in the Scheme 1. Diacetone D-glucose (1) prepared from $\mathrm{D}-(+)$-glucose by treating with acetone in the presence of a catalytic amount of sulphuric acid according to the literature procedure, ${ }^{49}$ reduction of 2 (prepared by Swern oxidation of 1 ) with $\mathrm{NaBH}_{4}$ in aq. ethanol at $0{ }^{\circ} \mathrm{C}$ for $1 \mathrm{~h}$ gave 3 (77\%), which on subsequent propargylation in DMF in the presence of $\mathrm{NaH}$ for $1 \mathrm{~h}$ afforded propargyl ether $4(80 \%)$. Now the propargyl ether converted into triazole $5(82 \%)$ by using 1,3-dipolar cycloaddition with p-chlorophenyl azide was carried out at ambient temperature in the presence of $\mathrm{CuSO}_{4}$ and sodium ascorbate in a mixture of $1: 1 t$ - $\mathrm{BuOH}-\mathrm{H}_{2} \mathrm{O}$ as reported by Sharpless. Acid hydrolysis of 5,6-acetonide 5 in $60 \%$ AcOH furnished the diol 6 (85\%), which on oxidative cleavage with $\mathrm{NaIO}_{4}$ gave the aldehyde 7 . Subsequently one-pot synthesis of triazole linked to thiazolidenone glycosides was carried out by the condensation reaction between 7 , primary aromatic amine and a thioglycolic acid in the presence of $\mathrm{ZnCl}_{2}$ under microwave irradiation/conventional heating (Scheme 2 ). In the classical method, the reactions were performed in dry toluene at reflux for a long time $(2-4 \mathrm{~h})$, often leading to degradation processes and consequent low yields of isolated products, whereas the application of microwave assisted technology, the reaction is completed in only 5-10 minutes and the compounds, isolated by conventional work-up, are obtained in satisfactory yields, often higher

Table 1. Synthesis of compounds $10 a-r$

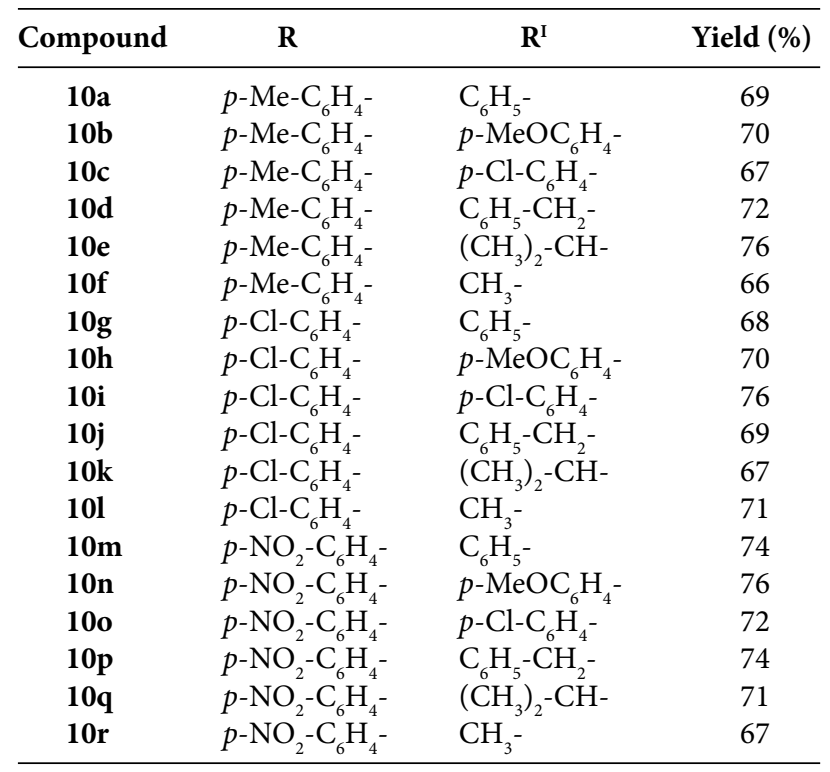




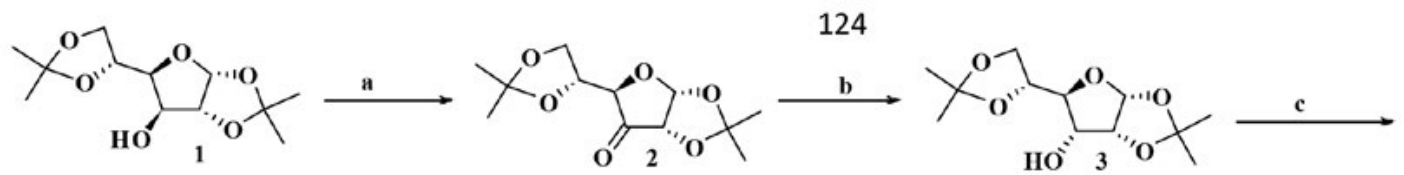<smiles>C#CCO[C@H]1[C@H]([C@H]2COC(C)(C)O2)O[C@H]2OC(C)(CCC(C)C)O[C@@H]21</smiles>

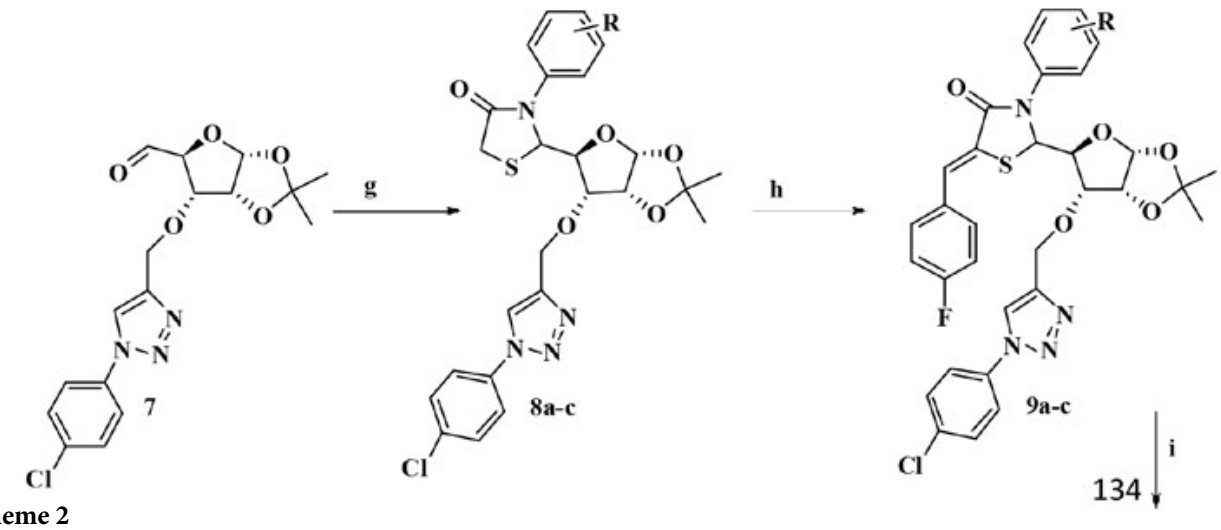

$\mathrm{R}=\mathrm{a}) 4-\mathrm{CH}_{3}-\mathrm{C}_{6} \mathrm{H}_{5}$ b) $4-\mathrm{Cl}-\mathrm{C}_{6} \mathrm{H}_{5}$; c) $4-\mathrm{NO}_{2}-\mathrm{C}_{6} \mathrm{H}_{5}$

Reagents and conditions: a) $\mathrm{COCl}_{2}, \mathrm{CH}_{2} \mathrm{Cl}_{2}, \mathrm{Et}_{3} \mathrm{~N},-78^{\circ} \mathrm{C}$ $\rightarrow \mathrm{rt}, 1.5 \mathrm{~h}, 83 \%$; b) $\mathrm{NaBH}_{4}, \mathrm{EtOH}, \mathrm{H}_{2} \mathrm{O},(19: 1), 0{ }^{\circ} \mathrm{C} \rightarrow \mathrm{rt}$, $78 \%$; c) Propargyl bromide, NaH, DMF, $0{ }^{\circ} \mathrm{C} \rightarrow \mathrm{rt}$; d) $p$-Chlorophenylazide, sodium ascorbate, $\mathrm{CuSO}_{4} \cdot 5 \mathrm{H}_{2} \mathrm{O}$, $t-\mathrm{BuOH} / \mathrm{H}_{2} \mathrm{O}, 0{ }^{\circ} \mathrm{C} \rightarrow \mathrm{rt}, 75 \%$; e) $60 \%$, AcOH, rt, 69\%; f) $\mathrm{NaIO}_{4}, \mathrm{CH}_{2} \mathrm{Cl}_{2}, 0^{\circ} \mathrm{C} \rightarrow \mathrm{rt}, 75 \%$; g) $\mathrm{Ar}-\mathrm{NH}_{2}, \mathrm{SHCH}_{2} \mathrm{COOH}$, $\mathrm{ZnCl}_{2}$, toluene, $80^{\circ} \mathrm{C}$, 85\%; h) $4-\mathrm{F}-\mathrm{C}_{6} \mathrm{H}_{4}-\mathrm{CHO}, \mathrm{AcOH} /$ $\mathrm{NaOAc}$, reflux, 82-88\%; i) R'- $\mathrm{NHNH}_{2} \cdot \mathrm{HCl}, \mathrm{AcOH} /$ $\mathrm{NaOAc}$ reflux, 76-67\%.

than those achieved by traditional methods. ${ }^{35}$ Compound 8 was then reacted with $p$-fluorobenzaldehyde in the presence of anhydrous $\mathrm{NaOAc}$ in glacial $\mathrm{AcOH}$ at reflux temperature to give chalcone derivatives of triazole linked thiazolidenone glycosides 9. Furthermore, the compounds upon cyclocondensation with alkyl/aryl hydrazines in the presence of anhydrous $\mathrm{NaOAc}$ in glacial $\mathrm{AcOH}$ at reflux temperature gave 10a-r in good yields. The versatility of the reaction is demonstrated by the fact that both aromatic hydrazines, such as phenyl hydrazines, and aliphatic hydrazines, such as benzylhydrazine, isopropylhydrazine, methylhydrazine, afforded their corresponding 10a-r in good yields. The structures of synthesized compounds

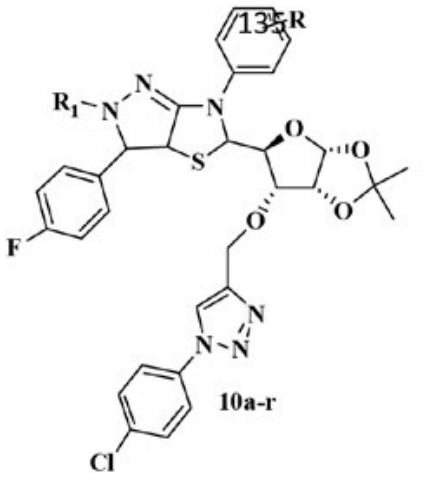

were confirmed by IR, NMR, MS and elemental analysis. Furthermore, the compounds were subjected to nematicidal and anti bacterial testing.

\section{Antifungal Activity}

The newly prepared compounds 10a-r were screened for their antifungal activity against four fungal organisms, viz Candida albicans (ATCC 10231), Aspergillus fumigates (HIC 6094), Trichophyton rubrum (IFO 9185) and Trichophyton mentagrophytes (IFO 40996) in dimethyl sulfoxide (DMSO) by agar diffusion method..$^{50}$ Amphotericin $\mathrm{B}$ was 
used as the standard drug and the zones of fungal inhibition values are reported in Table 3. In addition, the MIC and MFC values determined by the broth dilution method $^{51}$ are recorded in Table 4.

\section{Nematicidal Activity}

The compounds synthesized $\mathbf{1 0 a}-\mathbf{g}$ in this study were also screened for their nematicidal activity against Dietylenchus myceliophagus and Caenorhabditis elegans by aqueous in vitro screening technique ${ }^{52}$ at various concentrations. The nematicidal activity of each tested compound was compared with the standard drug Levamisole. The results have been expressed in terms of $\mathrm{LD}_{50}$ i.e. median le- thal dose at which $50 \%$ of nematodes became immobile (dead). The screened data reveal that compounds 10e and $10 f$ are the most effective against Dietylenchus myceliophagus and Caenorhabditis elegans with $\mathrm{LD}_{50} 190$ and $220 \times$ $10^{-6}$ respectively, whereas the other tested compounds showed moderate activity. The $\mathrm{LD}_{50}$ values of the compounds screened are presented in Table 2.

\section{Experimental}

Commercial grade reagents were used as supplied. Solvents except analytical reagent grade were dried and purified according to literature when necessary. Reaction progress and purity of the compounds were checked by

Table 2. Median lethal dose $\mathrm{LD}_{50}(\mathrm{ppm})$ of compounds $10 \mathbf{a}-\mathbf{r}$

\begin{tabular}{cccccc}
\hline Compd. & $\begin{array}{c}\text { D. mycelio- } \\
\text { phagus }\end{array}$ & C. elegans & $\begin{array}{c}\text { Compd. } \\
\text { phagus }\end{array}$ & D. mycelio- & C. elegans \\
\hline $\mathbf{1 0 a}$ & 850 & 670 & $\mathbf{1 0 j}$ & 180 & 200 \\
$\mathbf{1 0 b}$ & 950 & 870 & $\mathbf{1 0 k}$ & 520 & 670 \\
$\mathbf{1 0 c}$ & 240 & 360 & $\mathbf{1 0 l}$ & 190 & 210 \\
$\mathbf{1 0 d}$ & 190 & 210 & $\mathbf{1 0 m}$ & 800 & 620 \\
$\mathbf{1 0}$ & 550 & 600 & $\mathbf{1 0 n}$ & 920 & 810 \\
$\mathbf{1 0 f}$ & 160 & 200 & $\mathbf{1 0 0}$ & 230 & 350 \\
$\mathbf{1 0 g}$ & 810 & 650 & $\mathbf{1 0 p}$ & 190 & 190 \\
$\mathbf{1 0 h}$ & 900 & 820 & $\mathbf{1 0 q}$ & 510 & 600 \\
$\mathbf{1 0 i}$ & 290 & 380 & $\mathbf{1 0 r}$ & 180 & 200 \\
Oxamyl & 150 & 180 & Oxamyl & 150 & 180 \\
\hline
\end{tabular}

$\mathrm{LD}_{50}$, median lethal dose (the concentration at which $50 \%$ nematodes became immobile)

Table 3. Inhibitory zone diameters $(\mathrm{mm})$ of $\mathbf{1 0 a}-\mathbf{r}$ against tested fungal strains

\begin{tabular}{|c|c|c|c|c|}
\hline \multirow{2}{*}{ Compound } & \multicolumn{4}{|c|}{ Mean zone of inhibition (MZI) in $\mathrm{mm}^{\mathrm{a}}$} \\
\hline & C. albicans & A. fumigatus & T. rubrum & T. mentagrophytes \\
\hline $10 \mathbf{a}$ & 11 & 10 & - & 09 \\
\hline $10 b$ & 09 & 15 & 13 & 12 \\
\hline $10 \mathrm{c}$ & 13 & 11 & 11 & 10 \\
\hline 10d & 22 & 16 & 15 & 16 \\
\hline $10 \mathrm{e}$ & - & 13 & - & - \\
\hline $10 f$ & 20 & 20 & 20 & 18 \\
\hline $10 \mathrm{~g}$ & - & - & 17 & - \\
\hline $10 \mathrm{~h}$ & 14 & - & - & - \\
\hline $10 \mathbf{i}$ & - & - & 15 & - \\
\hline $10 \mathrm{j}$ & 21 & 17 & 14 & 16 \\
\hline $10 \mathbf{k}$ & 10 & - & 11 & 12 \\
\hline 101 & 18 & 15 & 13 & 16 \\
\hline $10 \mathrm{~m}$ & 08 & 11 & 10 & 09 \\
\hline $10 n$ & 13 & - & - & - \\
\hline $10 \mathrm{o}$ & 15 & - & - & - \\
\hline $10 p$ & 19 & 16 & 11 & 13 \\
\hline $10 q$ & - & - & 09 & - \\
\hline $10 \mathrm{r}$ & 21 & 14 & 15 & 14 \\
\hline Amphotericin B & 25 & 20 & 20 & 18 \\
\hline
\end{tabular}

Amphotericin B (100 $\mu \mathrm{g} / \mathrm{disc})$ was used as the positive reference; compounds 10a-r $(300 \mu \mathrm{g} / \mathrm{disc})$.

- indicates no sensitivity or MZI lower that $7 \mathrm{~mm}$. ${ }^{a}$ Values are mean $(\mathrm{n}=3)$. 
Table 4. Minimum inhibitory concentration (MIC) and minimum fungicidal concentration (MFC) in $\mu \mathrm{g} / \mathrm{mL}$ of compounds 10a-r

\begin{tabular}{|c|c|c|c|c|c|c|c|c|}
\hline \multirow{2}{*}{ Compd. } & \multicolumn{2}{|c|}{ C. albicans } & \multicolumn{2}{|c|}{ A. fumigatus } & \multicolumn{2}{|c|}{ T. rubrum } & \multicolumn{2}{|c|}{ T. mentagrophytes } \\
\hline & MIC & MFC & MIC & MFC & MIC & MFC & MIC & MFC \\
\hline $10 a$ & - & - & 25.0 & 25.0 & 25.0 & 50.0 & 25.0 & 25.0 \\
\hline $10 \mathrm{~b}$ & 25.0 & 25.0 & 25.0 & 50.0 & 12.5 & 12.5 & 12.5 & 25.0 \\
\hline $10 \mathrm{c}$ & 12.5 & 25.0 & 6.25 & 12.5 & 6.25 & 12.5 & 6.25 & 12.5 \\
\hline 10d & 3.12 & 6.25 & 25.0 & 25.0 & 12.5 & 25.0 & 25.0 & 25.0 \\
\hline $10 \mathrm{e}$ & 12.5 & 25.0 & - & - & - & - & - & - \\
\hline $10 \mathrm{f}$ & 3.12 & 3.12 & 3.12 & 6.25 & 3.12 & 6.25 & 6.25 & 12.5 \\
\hline $10 \mathrm{~g}$ & 6.25 & 6.25 & 12.5 & 25.0 & 12.5 & 12.5 & 6.25 & 12.5 \\
\hline $10 \mathrm{~h}$ & 25.0 & 50.0 & - & - & 25.0 & 50.0 & 25.0 & 50.0 \\
\hline $10 \mathrm{i}$ & 12.5 & 12.5 & 25.0 & 25.0 & 12.5 & 12.5 & 25.0 & 50.0 \\
\hline $10 j$ & 3.12 & 6.25 & 6.25 & 12.5 & 6.25 & 12.5 & 12.5 & 25.0 \\
\hline $10 \mathrm{k}$ & 12.5 & 25.0 & 25.0 & 25.0 & - & - & 25.0 & 50.0 \\
\hline 101 & 12.5 & 25.0 & 6.25 & 50.0 & 6.25 & 25.0 & - & - \\
\hline $10 \mathrm{~m}$ & 12.5 & 25.0 & 12.5 & 50.0 & 6.25 & 25.0 & 12.5 & 12.5 \\
\hline $10 n$ & 6.25 & 12.5 & 12.5 & 25.0 & 12.5 & 25.0 & - & - \\
\hline $10 \mathrm{o}$ & 25.0 & 25.0 & 25.0 & 50.0 & 25.0 & 50.0 & 6.25 & 12.5 \\
\hline $10 p$ & 12.5 & 25.0 & - & - & - & - & - & - \\
\hline $10 q$ & 25.0 & 25.0 & 25.0 & 50.0 & 12.5 & 25.0 & 12.5 & 25.0 \\
\hline $10 \mathrm{r}$ & 12.5 & 25.0 & 12.5 & 25.0 & 12.5 & 25.0 & 12.5 & 25.0 \\
\hline Amphotericin B & 6.25 & 12.5 & 3.12 & 6.25 & 3.12 & 12.5 & 3.12 & 12.5 \\
\hline
\end{tabular}

- Indicates fungi are resistant to the compound $>100 \mu \mathrm{g} / \mathrm{mL}$ concentration.

thin-layer chromatography (TLC) on pre-coated silica gel F254 plates from Merck and compounds were visualized either by exposure to UV light or dipping in $1 \%$ aqueous potassium permanganate solution. Silica gel chromatographic columns (60-120 mesh) were used for separations. Microwave reactions were carried out in mini lab microwave catalytic reactor (ZZKD, WBFY-201). Optical rotations were measured on Perkin-Elmer 141 polarimeter by using a 2 $\mathrm{mL}$ cell with a path length of $1 \mathrm{dm}$ with $\mathrm{CHCl}_{3}$ or $\mathrm{CDCl}_{3}$ as solvent. All melting points are uncorrected and measured using Fisher-Johns apparatus. IR spectra were recorded as $\mathrm{KBr}$ disks on a Perkin-Elmer FT IR spectrometer. The ${ }^{1} \mathrm{H}$ NMR and ${ }^{13} \mathrm{C}$ NMR spectra were recorded on a Varian Gemini spectrometer $\left(300 \mathrm{MHz}\right.$ for ${ }^{1} \mathrm{H}$ and $75 \mathrm{MHz}$ for ${ }^{13} \mathrm{C}$ ). Chemical shifts are reported as $\delta$ on ppm scale against TMS as the internal reference and coupling constants $(J)$ are reported in $\mathrm{Hz}$ units. Mass spectra were recorded on a VG micro mass $7070 \mathrm{H}$ spectrometer. Elemental analyses $(\mathrm{C}, \mathrm{H}$, N) were determined by a Perkin-Elmer $240 \mathrm{CHN}$ elemental analyzer and are within $\pm 0.4 \%$ of theoretical values.

2-((3aR,5S,6S,6aR)-6-((1-(4-Chlorophenyl)-1H-1,2,3triazol-4-yl)methoxy)-2,2-dimethyltetrahydrofuro[2,3-d][1,3] dioxol-5-yl)-3-phenylthiazolidin-4-ones 8a-c. To a solution of diol $6(0.200 \mathrm{~g}, 0.48 \mathrm{mmol})$ in $\mathrm{CH}_{2} \mathrm{Cl}_{2}(5 \mathrm{~mL}), \mathrm{NaIO}_{4}(0.130 \mathrm{~g}, 0.61 \mathrm{mmol})$ was added at 0 ${ }^{\circ} \mathrm{C}$ and stirred at room temperature for $6 \mathrm{~h}$. The reaction mixture was filtered and washed with $\mathrm{CH}_{2} \mathrm{Cl}_{2}(2 \times 10 \mathrm{~mL})$. It was dried $\left(\mathrm{Na}_{2} \mathrm{SO}_{4}\right)$ and evaporated to give aldehyde 7 $(0.150 \mathrm{~g})$ in quantitative yield as a yellow liquid, which was used as such for the next reaction.
To a stirred mixture of 7 (0.150 g, $0.395 \mathrm{mmol})$, aromatic amine $(0.395 \mathrm{mmol})$ and anhydrous thioglycolic acid $(0.160 \mathrm{~g}, 0.211 \mathrm{mmol})$ in dry toluene $(5 \mathrm{~mL}), \mathrm{ZnCl}_{2}$ $(0.100 \mathrm{~g}, 0.751 \mathrm{mmol})$ was added after $2 \mathrm{~min}$ and irradiated in microwave bath reactor at $280 \mathrm{~W}$ for $4-7$ minutes at $110^{\circ} \mathrm{C}$. After cooling, the filtrate was concentrated to dryness under reduced pressure and the residue was taken-up in ethyl acetate. The ethyl acetate layer was washed with $5 \%$ sodium bicarbonate solution and finally with brine. The organic layer was dried over $\mathrm{Na}_{2} \mathrm{SO}_{4}$ and evaporated to dryness at reduced pressure. The crude product thus obtained was purified by column chromatography on silica gel (60-120 mesh) with hexane/ethyl acetate as the eluent.

2-((3aR,5S,6S,6aR)-6-((1-(4-Chlorophenyl)-1H-1,2,3triazol-4-yl)methoxy)-2,2-dimethyltetrahydrofuro[2,3-d][1,3] dioxol-5-yl)-3-p-tolylthiazolidin-4-one (8a) m.p. $191-193^{\circ} \mathrm{C}$; ${ }^{1} \mathrm{H}$ NMR $\left(300 \mathrm{MHz}, \mathrm{CDCl}_{3}\right) \delta 8.26$ $(\mathrm{d}, J=8.7 \mathrm{~Hz}, 2 \mathrm{H}, \mathrm{Ar}-\mathrm{H}), 8.04(\mathrm{~s}, 1 \mathrm{H}, \mathrm{Ar}-\mathrm{H}), 7.54(\mathrm{~d}, J=9.2$ $\mathrm{Hz}, 2 \mathrm{H}, \mathrm{Ar}-\mathrm{H}), 7.39$ (d, $J=8.33 \mathrm{~Hz}, 2 \mathrm{H}, \mathrm{Ar}-\mathrm{H}), 7.15$ (d, $J=$ $8.3 \mathrm{~Hz}, 2 \mathrm{H}, \mathrm{Ar}-\mathrm{H}), 5.76\left(\mathrm{~d}, J=3.6 \mathrm{~Hz}, 1 \mathrm{H}, \mathrm{C}_{1} \mathrm{H}\right), 4.96(\mathrm{~d}, J$ $=5.2 \mathrm{~Hz}, 1 \mathrm{H}, \mathrm{CH}-\mathrm{S}), 4.66\left(\mathrm{t}, J=3.9 \mathrm{~Hz}, 1 \mathrm{H}, \mathrm{C}_{2} \mathrm{H}\right), 4.54(\mathrm{~s}$, $\left.2 \mathrm{H}, \mathrm{OCH}_{2}\right), 3.96-3.91\left(\mathrm{~m}, 1 \mathrm{H}, \mathrm{C}_{4} \mathrm{H}\right), 3.76\left(\mathrm{~s}, 2 \mathrm{H}, \mathrm{CH}_{2}\right)$, $3.26\left(\mathrm{dd}, J_{1}=9.1 \mathrm{~Hz}, J_{2}=4.2 \mathrm{~Hz}, 1 \mathrm{H}, \mathrm{C}_{3} \mathrm{H}\right), 2.3\left(\mathrm{~s}, 3 \mathrm{H}, \mathrm{CH}_{3}\right)$, $1.53\left(\mathrm{~s}, 3 \mathrm{H}, \mathrm{CH}_{3}\right), 1.36\left(\mathrm{~m}, 3 \mathrm{H}, \mathrm{CH}_{3}\right) ;{ }^{13} \mathrm{C} \mathrm{NMR}(75 \mathrm{MHz}$, $\left.\mathrm{CDCl}_{3}\right) \delta 172.6,143.2,137.4,133.6,132.3,131.2,128.4$, $127.9,124.8,122.9,119.2,111.2,103.8,81.2,78.1,74.1$, 65.9, 51.4, 26.1, 16.1; MS: $m / z\left(\mathrm{M}^{+}+\mathrm{Na}\right)$ 565. Anal. Calcd for $\mathrm{C}_{26} \mathrm{H}_{27} \mathrm{ClN}_{4} \mathrm{O}_{5} \mathrm{~S}: \mathrm{C}, 57.51 ; \mathrm{H}, 5.51 ; \mathrm{N}, 10.32$. Found: C, $57.32 ; \mathrm{H}, 5.35 ; \mathrm{N}, 10.09$. 
3-(4-Chlorophenyl)-2-((3aR,5S,6S,6aR)-6-((1-(4-chlorophenyl)-1H-imidazol-4-yl)methoxy)-2,2-dimethyltetrahydrofuro $[2,3-d][1,3]$ dioxol-5-yl)thiazolidin-4-one (8b) m.p. $216-218{ }^{\circ} \mathrm{C}$; ${ }^{1} \mathrm{H}$ NMR $(300 \mathrm{MHz}$, $\left.\mathrm{CDCl}_{3}\right) \delta 8.02(\mathrm{~s}, 1 \mathrm{H}, \mathrm{Ar}-\mathrm{H}), 7.50(\mathrm{~d}, J=9.2 \mathrm{~Hz}, 4 \mathrm{H}, \mathrm{Ar}-\mathrm{H})$, $7.41(\mathrm{~d}, J=8.9 \mathrm{~Hz}, 4 \mathrm{H}, \mathrm{Ar}-\mathrm{H}), 5.72\left(\mathrm{~d}, J=3.6 \mathrm{~Hz}, 1 \mathrm{H}, \mathrm{C}_{1} \mathrm{H}\right)$, $4.94(\mathrm{~d}, J=5.2 \mathrm{~Hz}, \mathrm{CH}-\mathrm{S}), 4.60\left(\mathrm{t}, J=3.9 \mathrm{~Hz}, 1 \mathrm{H}, \mathrm{C}_{2} \mathrm{H}\right), 4.51$ (s, $\left.2 \mathrm{H}, \mathrm{OCH}_{2}\right), 3.96-3.91\left(\mathrm{~m}, 1 \mathrm{H}, \mathrm{C}_{4} \mathrm{H}\right), 3.76\left(\mathrm{~s}, 2 \mathrm{H}, \mathrm{CH}_{2}\right)$, $3.31\left(\mathrm{dd}, J_{1}=9.1 \mathrm{~Hz}, J_{2}=4.2 \mathrm{~Hz}, 1 \mathrm{H}, \mathrm{C}_{3} \mathrm{H}\right), 1.55(\mathrm{~s}, 3 \mathrm{H}$, $\left.\mathrm{CH}_{3}\right), 1.32\left(\mathrm{~m}, 3 \mathrm{H}, \mathrm{CH}_{3}\right) ;{ }^{13} \mathrm{C}$ NMR $\left(75 \mathrm{MHz}, \mathrm{CDCl}_{3}\right) \delta$ 170.6, 139.4, 134.8, 133.2, 129.4, 128.6, 125.6, 122.2, 119.4, $111.2,104.9,81.5,74.5,66.3,52.6,34.6,26.5$; MS: $m / z$ $\left(\mathrm{M}^{+}+\mathrm{H}\right)$ 563. Anal. Calcd for $\mathrm{C}_{25} \mathrm{H}_{24} \mathrm{Cl}_{2} \mathrm{~N}_{4} \mathrm{O}_{5} \mathrm{~S}: \mathrm{C}, 53.29 ; \mathrm{H}$, 4.29 ; N, 9.94. Found: C, 53.21; H, 4.16; N, 9.83.

2-((3aR,5S,6S,6aR)-6-((1-(4-Chlorophenyl)-1H-1,2,3triazol-4-yl)methoxy)-2,2-dimethyltetrahydrofuro[2,3-d][1,3] dioxol-5-yl)-3-(4-nitrophenyl)thiazolidin-4-one (8c) m.p. 201-205 ${ }^{\circ} \mathrm{C}$; ${ }^{1} \mathrm{H}$ NMR $(300 \mathrm{MHz}$, $\left.\mathrm{CDCl}_{3}\right) \delta 8.26(\mathrm{~d}, J=8.7 \mathrm{~Hz}, 2 \mathrm{H}), 8.04(\mathrm{~s}, 1 \mathrm{H}, \mathrm{Ar}-\mathrm{H}), 7.51$ $(\mathrm{d}, J=9.2 \mathrm{~Hz}, 2 \mathrm{H}, \mathrm{Ar}-\mathrm{H}), 7.42(\mathrm{~d}, J=8.5 \mathrm{~Hz}, 2 \mathrm{H}, \mathrm{Ar}-\mathrm{H})$, $6.82(\mathrm{~d}, J=9.8 \mathrm{~Hz}, 2 \mathrm{H}, \operatorname{Ar}-\mathrm{H}), 5.71(\mathrm{~d}, J=3.6 \mathrm{~Hz}, 1 \mathrm{H}$, $\left.\mathrm{C}_{1} \mathrm{H}\right), 4.96(\mathrm{~d}, J=5.2 \mathrm{~Hz}, \mathrm{CH}-\mathrm{S}), 4.62(\mathrm{t}, J=3.9 \mathrm{~Hz}, 1 \mathrm{H}$, $\left.\mathrm{C}_{2} \mathrm{H}\right), 4.53\left(\mathrm{~s}, 2 \mathrm{H}, \mathrm{OCH}_{2}\right), 3.96-3.91\left(\mathrm{~m}, 1 \mathrm{H}, \mathrm{C}_{4} \mathrm{H}\right), 3.76(\mathrm{~s}$, $\left.2 \mathrm{H}, \mathrm{CH}_{2}\right), 3.28\left(\mathrm{dd}, J_{1}=9.1 \mathrm{~Hz}, J_{2}=4.2 \mathrm{~Hz}, 1 \mathrm{H}, \mathrm{C}_{3} \mathrm{H}\right), 1.52$ $\left(\mathrm{s}, 3 \mathrm{H}, \mathrm{CH}_{3}\right), 1.34\left(\mathrm{~m}, 3 \mathrm{H}, \mathrm{CH}_{3}\right) ;{ }^{12} \mathrm{C} \mathrm{NMR}\left(75 \mathrm{MHz}, \mathrm{CDCl}_{3}\right)$ $\delta 170.6,147.5,144.4,143.2,134.8,131.2,128.6,124.6$, $122.4,119.8,111.8,104.9,81.5,78.2,74.8,66.9,52.4$, 34.6, 26.8; MS: $m / z\left(\mathrm{M}^{+}+\mathrm{H}\right)$ 574. Anal. Calcd for $\mathrm{C}_{25} \mathrm{H}_{24} \mathrm{ClN}_{5} \mathrm{O}_{7} \mathrm{~S}$ : C, 52.31; H, 4.21; N, 12.20. Found: C, 52.26; $\mathrm{H}, 4.19 ; \mathrm{N}, 12.11$.

(Z)-5-Benzylidene-2-((3aR,5S,6S,6aR)-6-((1-(4-chlorophenyl)-1H-1,2,3-triazol-4-yl)methoxy)-2,2-dimethyltetrahydrofuro $[2,3-d][1,3]$ dioxol-5-yl)-3-phenylthiazolidin-4-ones 9a-c. A mixture of compound 8 (0.01 $\mathrm{mol}), p$-fluorobenzaldehyde $(0.02 \mathrm{~mol})$ and sodium acetate $(0.01 \mathrm{~mol})$ in anhydrous glacial acetic acid $(20 \mathrm{~mL})$, was refluxed for $3 \mathrm{~h}$. The reaction mixture was concentrated and then poured into ice cold water, the solid thus separated was filtered, washed with water and crystallized from glacial acetic acid to afford pure compounds.

(Z)-2-((3aR,5S,6S,6aR)-6-((1-(4-Chlorophenyl)-1H1,2,3-triazol-4-yl)methoxy)-2,2-dimethyltetrahydrofuro[2,3-d] [1,3]dioxol-5-yl)-5-(4-fluorobenzylidene)-3p-tolylthiazolidin-4-one (9a). This compound was obtained as brown solid, m.p. $231-235^{\circ} \mathrm{C}$; ${ }^{1} \mathrm{H}$ NMR $(300$ $\left.\mathrm{MHz}, \mathrm{CDCl}_{3}\right) \delta 8.22(\mathrm{~d}, J=8.7 \mathrm{~Hz}, 2 \mathrm{H}, \mathrm{Ar}-\mathrm{H}), 8.06(\mathrm{~s}, 1 \mathrm{H}$, $\mathrm{Ar}-\mathrm{H}), 7.84(\mathrm{~s}, 1 \mathrm{H}, \mathrm{CH}=\mathrm{C}), 7.54(\mathrm{~d}, J=9.2 \mathrm{~Hz}, 2 \mathrm{H}, \mathrm{Ar}-\mathrm{H})$, $7.39(\mathrm{~d}, J=8.33 \mathrm{~Hz}, 2 \mathrm{H}, \mathrm{Ar}-\mathrm{H}), 7.15(\mathrm{~d}, J=8.3 \mathrm{~Hz}, 2 \mathrm{H}, \mathrm{Ar}-$ $\mathrm{H}), 6.91-6.87(\mathrm{~m}, 5 \mathrm{H}, \mathrm{Ar}-\mathrm{H}$ and $\mathrm{CH}-\mathrm{S}), 5.76(\mathrm{~d}, J=3.6 \mathrm{~Hz}$, $\left.1 \mathrm{H}, \mathrm{C}_{1} \mathrm{H}\right), 4.66\left(\mathrm{t}, J=3.9 \mathrm{~Hz}, 1 \mathrm{H}, \mathrm{C}_{2} \mathrm{H}\right), 4.54\left(\mathrm{~s}, 2 \mathrm{H}, \mathrm{OCH}_{2}\right)$, $3.96-3.91\left(\mathrm{~m}, 1 \mathrm{H}, \mathrm{C}_{4} \mathrm{H}\right), 3.26\left(\mathrm{dd}, J_{1}=9.1 \mathrm{~Hz}, J_{2}=4.2 \mathrm{~Hz}\right.$, $\left.1 \mathrm{H}, \mathrm{C}_{3} \mathrm{H}\right), 2.3\left(\mathrm{~s}, 3 \mathrm{H}, \mathrm{CH}_{3}\right), 1.53\left(\mathrm{~s}, 3 \mathrm{H}_{1} \mathrm{CH}_{3}\right), 1.36(\mathrm{~m}$, $\left.3 \mathrm{H}, \mathrm{CH}_{3}\right) ;{ }^{13} \mathrm{C} \mathrm{NMR}\left(75 \mathrm{MHz}, \mathrm{CDCl}_{3}\right) \delta 172.6,143.2,137.4$,
$133.6,132.3,131.2,128.4,127.9,124.8,122.9,119.2,111.2$, 103.8, 81.2, 78.1, 74.1, 65.9, 51.4, 26.1, 16.1; MS: $\mathrm{m} / \mathrm{z}$ $\left(\mathrm{M}^{+}+\mathrm{H}\right)$ 649. Anal. Calcd for $\mathrm{C}_{33} \mathrm{H}_{30} \mathrm{ClFN}_{4} \mathrm{O}_{5} \mathrm{~S}: \mathrm{C}, 61.06 ; \mathrm{H}$, $4.66 ; \mathrm{N}, 8.63$. Found: C, 60.82; H, 4.45; N, 8.43.

(Z)-3-(4-Chlorophenyl)-2-((3aR,5S,6S,6aR)-6-((1-(4chlorophenyl)-1H-1,2,3-triazol-4-yl)methoxy)-2,2-dimethyltetrahydrofuro[2,3-d] [1,3] dioxol-5-yl)-5-(4-fluorobenzylidene)thiazolidin-4-one (9b). The compound was obtained as dark yellow solid, m.p. $232-235{ }^{\circ} \mathrm{C} ;{ }^{1} \mathrm{H}$ NMR $\left(300 \mathrm{MHz}, \mathrm{DMSO}-d_{6}\right) \delta 8.06(\mathrm{~s}, 1 \mathrm{H}, \mathrm{Ar}-\mathrm{H}), 7.82$ (s, $1 \mathrm{H}, \mathrm{CH}=\mathrm{C}), 7.52(\mathrm{~d}, J=9.2 \mathrm{~Hz}, 4 \mathrm{H}, \mathrm{Ar}-\mathrm{H}), 7.43(\mathrm{~d}, J=8.9$ $\mathrm{Hz}, 4 \mathrm{H}, \mathrm{Ar}-\mathrm{H}), 6.91-6.87$ (m, 5H, Ar-H and CH-S), 5.72 $\left(\mathrm{d}, J=3.6 \mathrm{~Hz}, 1 \mathrm{H}, \mathrm{C}_{1} \mathrm{H}\right), 4.60\left(\mathrm{t}, J=3.9 \mathrm{~Hz}, 1 \mathrm{H}, \mathrm{C}_{2} \mathrm{H}\right), 4.51$ (s, $\left.2 \mathrm{H}, \mathrm{OCH}_{2}\right), 3.96-3.91\left(\mathrm{~m}, 1 \mathrm{H}, \mathrm{C}_{4} \mathrm{H}\right), 3.31\left(\mathrm{dd}, J_{1}=9.1\right.$ $\left.\mathrm{Hz}, \mathrm{J}_{2}=4.2 \mathrm{~Hz}, 1 \mathrm{H}, \mathrm{C}_{3} \mathrm{H}\right), 1.55\left(\mathrm{~s}, 3 \mathrm{H}, \mathrm{CH}_{3}\right), 1.32(\mathrm{~m}, 3 \mathrm{H}$, $\left.\mathrm{CH}_{3}\right) ;{ }^{13} \mathrm{C}$ NMR (75 MHz, DMSO- $\left.d_{6}\right) \delta 170.6,138.4,134.8$, 133.2, 130.8, 129.4, 128.6, 124.6, 122.2, 119.4, 111.2, 104.9, 81.5, 74.5, 66.3, 52.6, 34.6, 26.5; MS: $m / z\left(\mathrm{M}^{+}+\mathrm{H}\right) 669$. Anal. Calcd for $\mathrm{C}_{32} \mathrm{H}_{27} \mathrm{Cl}_{2} \mathrm{FN}_{4} \mathrm{O}_{5} \mathrm{~S}$ : C, 57.40; H, 4.06; N, 8.37. Found: C, $57.21 ; \mathrm{H}, 4.01 ; \mathrm{N}, 8.03$.

(Z)-2-((3aR,5S,6S,6aR)-6-((1-(4-Chlorophenyl)-1H1,2,3-triazol-4-yl)methoxy)-2,2-dimethyltetrahydrofuro[2,3-d][1,3] dioxol-5-yl)-5-(4-fluorobenzylidene)-3(4-nitrophenyl)thiazolidin-4-one (9c). The compound was obtained as brown solid, m.p. $216-218{ }^{\circ} \mathrm{C}$; ${ }^{1} \mathrm{H}$ NMR $\left(300 \mathrm{MHz}, \mathrm{DMSO}-d_{6}\right) \delta 8.26(\mathrm{~d}, J=8.7 \mathrm{~Hz}, 2 \mathrm{H}), 8.04(\mathrm{~s}, 1 \mathrm{H}$, $\mathrm{Ar}-\mathrm{H}), 7.84(\mathrm{~s}, 1 \mathrm{H}, \mathrm{CH}=\mathrm{C}), 7.51(\mathrm{~d}, J=9.2 \mathrm{~Hz}, 2 \mathrm{H}, \mathrm{Ar}-\mathrm{H})$, $7.42(\mathrm{~d}, J=8.5 \mathrm{~Hz}, 2 \mathrm{H}, \mathrm{Ar}-\mathrm{H}), 6.91-6.87(\mathrm{~m}, 5 \mathrm{H}, \mathrm{Ar}-\mathrm{H}$ and CH-S), $6.82(\mathrm{~d}, J=9.8 \mathrm{~Hz}, 2 \mathrm{H}, \mathrm{Ar}-\mathrm{H}), 5.71(\mathrm{~d}, J=3.6 \mathrm{~Hz}$, $\left.1 \mathrm{H}, \mathrm{C}_{1} \mathrm{H}\right), 4.62\left(\mathrm{t}, J=3.9 \mathrm{~Hz}, 1 \mathrm{H}, \mathrm{C}_{2} \mathrm{H}\right), 4.53\left(\mathrm{~s}, 2 \mathrm{H}, \mathrm{OCH}_{2}\right)$, 3.96-3.91 (m, $\left.1 \mathrm{H}, \mathrm{C}_{4} \mathrm{H}\right), 3.76\left(\mathrm{~s}, 2 \mathrm{H}, \mathrm{CH}_{2}\right), 3.28\left(\mathrm{dd}, J_{1}=9.1\right.$ $\left.\mathrm{Hz}, J_{2}=4.2 \mathrm{~Hz}, 1 \mathrm{H}, \mathrm{C}_{3} \mathrm{H}\right), 1.52\left(\mathrm{~s}, 3 \mathrm{H}, \mathrm{CH}_{3}\right), 1.34(\mathrm{~m}$, $\left.3 \mathrm{H}_{1} \mathrm{CH}_{3}\right) ;{ }^{13} \mathrm{C} \mathrm{NMR}\left(75 \mathrm{MHz}, \mathrm{CDCl}_{3}\right) \delta 170.6,147.5,144.4$, 143.2, 138.4, 134.8, 130.6, 131.2, 128.6, 124.6, 122.4, 119.8, $111.8,104.9,81.5,78.2,74.8,66.9,52.4,26.8$; MS: $\mathrm{m} / \mathrm{z}$ $\left(\mathrm{M}^{+}+\mathrm{Na}\right)$ 692. Anal. Calcd for $\mathrm{C}_{31} \mathrm{H}_{29} \mathrm{ClFN}_{5} \mathrm{O}_{7} \mathrm{~S}$ : C, 55.56; H, 4.36; N, 10.30. Found: C, 55.26; H, 4.29; N, 10.10.

5-((3aR,5S,6S,6aR)-6-((1-(4-Chlorophenyl)-1H-1,2,3triazol-4-yl)methoxy)-2,2-dimethyltetrahydrofuro[2,3-d][1,3] dioxol-5-yl)-3-(4-fluorophenyl)-2,6-diphenyl/ 2-alkyl-6-phenyl-3,3a,5,6-tetrahydro-2H-pyrazolo[3,4-d] thiazoles 10a-r. A mixture of compound 9 (5 $\mathrm{mmol})$, alkyl/aryl hydrazine $(5 \mathrm{mmol})$ and anhydrous sodium acetate $(5 \mathrm{mmol})$ in glacial acetic acid $(20 \mathrm{~mL})$, was refluxed for $7 \mathrm{~h}$. The reaction mixture was concentrated and cooled to room temperature, the solid thus separated was filtered, washed thoroughly with water. The crude product thus obtained was purified by column chromatography on silica gel with hexane/ethyl acetate as eluent to afford pure compounds.

5-((3aR,5S,6S,6aR)-6-((1-(4-Chlorophenyl)- $1 H-1,2,3-$ triazol-4-yl)methoxy)-2,2-dimethyltetrahydrofu- 
ro[2,3-d] $[1,3]$ dioxol-5-yl)-3-(4-fluorophenyl)-2-phenyl6-p-tolyl-3,3a,5,6-tetrahydro- $2 \mathrm{H}$-pyrazolo[3,4-d] thiazole (10a). This was obtained by reacting compound 9 a $(1 \mathrm{~g})$ and phenylhydrazine $(0.25 \mathrm{~g})$ as described in the typical procedure and isolated as a brown solid, yield $72 \%$, m.p. $248-250{ }^{\circ} \mathrm{C} ;{ }^{1} \mathrm{H}$ NMR $\left(300 \mathrm{MHz}\right.$, DMSO- $\left.d_{6}\right) \delta 8.20$ (d, $J=8.7 \mathrm{~Hz}, 2 \mathrm{H}, \mathrm{Ar}-\mathrm{H}), 8.04$ (s, $1 \mathrm{H}, \mathrm{Ar}-\mathrm{H}), 7.51$ (d, $J=9.2$ $\mathrm{Hz}, 2 \mathrm{H}, \mathrm{Ar}-\mathrm{H}$ ), 7.40 (s, $1 \mathrm{H}, \mathrm{CH}-\mathrm{S}), 7.29$ (d, $J=8.33 \mathrm{~Hz}, 2 \mathrm{H}$, Ar-H), 7.18-7.15 (m, 5H, Ar-H), $7.05(\mathrm{~d}, J=8.3 \mathrm{~Hz}, 2 \mathrm{H}$, Ar-H), 6.91-6.87 (m, 4H, Ar-H), $5.76(\mathrm{~d}, J=3.6 \mathrm{~Hz}, 1 \mathrm{H}$, $\left.\mathrm{C}_{1} \mathrm{H}\right), 5.62(\mathrm{~d}, J=2.2 \mathrm{~Hz}, 1 \mathrm{H}, \mathrm{S}-\mathrm{CH}), 5.25(\mathrm{~d}, J=2.2 \mathrm{~Hz}$, $1 \mathrm{H}, \mathrm{CH}-\mathrm{N}), 4.66\left(\mathrm{t}, J=3.9 \mathrm{~Hz}, 1 \mathrm{H}, \mathrm{C}_{2} \mathrm{H}\right), 4.54(\mathrm{~s}, 2 \mathrm{H}$, $\left.\mathrm{OCH}_{2}\right), 3.96-3.91\left(\mathrm{~m}, 1 \mathrm{H}, \mathrm{C}_{4} \mathrm{H}\right), 3.26\left(\mathrm{dd}, J_{1}=9.1 \mathrm{~Hz}, J_{2}=\right.$ $\left.4.2 \mathrm{~Hz}, 1 \mathrm{H}, \mathrm{C}_{3} \mathrm{H}\right), 2.3\left(\mathrm{~s}, 3 \mathrm{H}, \mathrm{CH}_{3}\right), 1.53\left(\mathrm{~s}, 3 \mathrm{H}_{1} \mathrm{CH}_{3}\right), 1.36$ $\left(\mathrm{m}, 3 \mathrm{H}, \mathrm{CH}_{3}\right) ;{ }^{13} \mathrm{C}$ NMR $\left(75 \mathrm{MHz}, \mathrm{DMSO}-d_{6}\right) \delta 172.6$, $143.2,141.4,137.4,133.6,132.3,131.2,129.3,125.3,128.4$, $127.9,124.8,122.9,119.2,111.2,103.8,81.2,78.1,74.1$, 65.9, 51.4, 26.1, 21.3, 16.1; MS: $m / z\left(\mathrm{M}^{+}+\mathrm{Na}\right)$ 761. Anal. Calcd for $\mathrm{C}_{39} \mathrm{H}_{36} \mathrm{ClFN}_{6} \mathrm{O}_{4} \mathrm{~S}: \mathrm{C}, 63.36 ; \mathrm{H}, 4.90 ; \mathrm{N}, 11.37$. Found: C, 63.12; H, 4.75; N, 11.29.

5-((3aR,5S,6S,6aR)-6-((1-(4-Chlorophenyl)-1H-1,2,3triazol-4-yl)methoxy)-2,2-dimethyltetrahydrofuro[2,3- $d][1,3]$ dioxol-5-yl)-3-(4-fluorophenyl)-2-(4-methoxyphenyl)-6-p-tolyl-3,3a,5,6-tetrahydro- $2 \mathrm{H}$-pyrazolo[3,4-d] thiazole (10 b). This was obtained by reacting compound $9 \mathbf{a}(1 \mathrm{~g})$ and $p$-methoxyphenylhydrazine $(0.35$ $\mathrm{g})$ as described in the typical procedure and isolated as a yellow solid, yield $62 \%$, m.p. $268-272{ }^{\circ} \mathrm{C} ;{ }^{1} \mathrm{H}$ NMR $(300$ $\left.\mathrm{MHz}, \mathrm{DMSO}-d_{6}\right) \delta 8.18(\mathrm{~d}, J=8.7 \mathrm{~Hz}, 2 \mathrm{H}, \mathrm{Ar}-\mathrm{H}), 8.04(\mathrm{~s}$, $1 \mathrm{H}, \operatorname{Ar}-\mathrm{H}), 7.51(\mathrm{~d}, J=9.2 \mathrm{~Hz}, 2 \mathrm{H}, \mathrm{Ar}-\mathrm{H}), 7.40(\mathrm{~s}, 1 \mathrm{H}$, CH-S), 7.29 (d, J=8.33 Hz, 2H, Ar-H), 7.12-7.10 (m, 4H, Ar-H), 7.05 (d, $J=8.3 \mathrm{~Hz}, 2 \mathrm{H}, \mathrm{Ar}-\mathrm{H}), 6.91-6.87(\mathrm{~m}, 4 \mathrm{H}$, Ar-H), $5.76\left(\mathrm{~d}, J=3.6 \mathrm{~Hz}, 1 \mathrm{H}, \mathrm{C}_{1} \mathrm{H}\right), 5.62(\mathrm{~d}, J=2.2 \mathrm{~Hz}, 1 \mathrm{H}$, $\mathrm{S}-\mathrm{CH}), 5.25(\mathrm{~d}, J=2.2 \mathrm{~Hz}, 1 \mathrm{H}, \mathrm{CH}-\mathrm{N}), 4.66(\mathrm{t}, J=3.9$ $\left.\mathrm{Hz}, 1 \mathrm{H}, \mathrm{C}_{2} \mathrm{H}\right), 4.54\left(\mathrm{~s}, 2 \mathrm{H}, \mathrm{OCH}_{2}\right), 3.96-3.94\left(\mathrm{~m}, 1 \mathrm{H}, \mathrm{C}_{4} \mathrm{H}\right)$, $3.91(\mathrm{~s}, 3 \mathrm{H}, \mathrm{OMe}), 3.26\left(\mathrm{dd}, J_{1}=9.1 \mathrm{~Hz}, J_{2}=4.2 \mathrm{~Hz}, 1 \mathrm{H}\right.$, $\left.\mathrm{C}_{3} \mathrm{H}\right), 2.3\left(\mathrm{~s}, 3 \mathrm{H}, \mathrm{CH}_{3}\right), 1.53\left(\mathrm{~s}, 3 \mathrm{H}, \mathrm{CH}_{3}\right), 1.36(\mathrm{~m}, 3 \mathrm{H}$, $\left.\mathrm{CH}_{3}\right) ;{ }^{13} \mathrm{C}$ NMR $\left(75 \mathrm{MHz}\right.$, DMSO- $\left.d_{6}\right) \delta 172.6,151.7,143.2$, 141.4, 137.4, 133.6, 132.3, 131.2, 129.3, 125.3, 128.4, 127.9, $124.8,122.9,119.2,111.2,103.8,81.2,78.1,74.1,65.9,55.8$, 51.4, 26.1, 21.3, 16.1; MS: $m / z\left(\mathrm{M}^{+}+\mathrm{H}\right) 789$. Anal. Calcd for $\mathrm{C}_{40} \mathrm{H}_{38} \mathrm{ClFN}_{6} \mathrm{O}_{5} \mathrm{~S}: \mathrm{C}, 62.46 ; \mathrm{H}, 4.98 ; \mathrm{N}, 10.92$. Found: C, 62.12; H, 4.88; N, 10.87 .

2-(4-Chlorophenyl)-5-((3aR,5S,6S,6aR)-6-((1-(4-chlorophenyl)-1H-1,2,3-triazol-4-yl)methoxy)-2,2-dimethyltetrahydrofuro[2,3-d] [1,3] dioxol-5-yl)-3-(4-fluorophenyl)-6-p-tolyl-3,3a,5,6-tetrahydro- $2 H$-pyrazolo[3,4- $\boldsymbol{d}]$ thiazole $(\mathbf{1 0 c})$. This was obtained by reacting compound $9 \mathbf{a}(1 \mathrm{~g})$ and $p$-chlorophenylhydrazine $(0.45 \mathrm{~g})$ as described in the typical procedure and isolated as a brown solid, yield $76 \%$, m.p. $248-251{ }^{\circ} \mathrm{C} ;{ }^{1} \mathrm{H}$ NMR (300 $\left.\mathrm{MHz}, \mathrm{DMSO}-d_{6}\right) \delta 8.21(\mathrm{~d}, J=8.7 \mathrm{~Hz}, 2 \mathrm{H}, \mathrm{Ar}-\mathrm{H}), 8.09$ (s,1H,Ar-H), 7.55 (d, J = 9.2 Hz, 2H, Ar-H), $7.42(\mathrm{~s}, 1 \mathrm{H}$, CH-S), 7.29 (d, J = $8.33 \mathrm{~Hz}, 2 \mathrm{H}, \mathrm{Ar}-\mathrm{H}), 7.10-7.08$ (m, 4H,
Ar-H), 7.05 (d, $J=8.3 \mathrm{~Hz}, 2 \mathrm{H}, \mathrm{Ar}-\mathrm{H}), 6.91-6.87(\mathrm{~m}, 4 \mathrm{H}$, $\operatorname{Ar}-\mathrm{H}), 5.76\left(\mathrm{~d}, J=3.6 \mathrm{~Hz}, 1 \mathrm{H}, \mathrm{C}_{1} \mathrm{H}\right), 5.62(\mathrm{~d}, J=2.2 \mathrm{~Hz}, 1 \mathrm{H}$, $\mathrm{S}-\mathrm{CH}), 5.25(\mathrm{~d}, J=2.2 \mathrm{~Hz}, 1 \mathrm{H}, \mathrm{CH}-\mathrm{N}), 4.66(\mathrm{t}, J=3.9 \mathrm{~Hz}$, $\left.1 \mathrm{H}, \mathrm{C}_{2} \mathrm{H}\right), 4.54\left(\mathrm{~s}, 2 \mathrm{H}, \mathrm{OCH}_{2}\right), 3.96-3.94\left(\mathrm{~m}, 1 \mathrm{H}, \mathrm{C}_{4} \mathrm{H}\right)$, $3.26\left(\mathrm{dd}, J_{1}=9.1 \mathrm{~Hz}, J_{2}=4.2 \mathrm{~Hz}, 1 \mathrm{H}, \mathrm{C}_{3} \mathrm{H}\right), 2.3\left(\mathrm{~s}, 3 \mathrm{H}_{1} \mathrm{CH}_{3}\right)$, $1.53\left(\mathrm{~s}, 3 \mathrm{H}, \mathrm{CH}_{3}\right), 1.36\left(\mathrm{~m}, 3 \mathrm{H}, \mathrm{CH}_{3}\right) ;{ }^{13} \mathrm{C} \mathrm{NMR}(75 \mathrm{MHz}$, DMSO- $\left.d_{6}\right) \delta 172.6,151.7,143.2,137.4,133.6,132.3,131.2$, $129.3,126.3,125.3,128.4,127.9,124.8,122.9,119.2,111.2$, 103.8, 81.2, 78.1, 74.1, 65.9, 51.4, 26.1, 21.3, 16.1; MS: $\mathrm{m} / \mathrm{z}$ $\left(\mathrm{M}^{+}+\mathrm{H}\right)$ 773. Anal. Calcd for $\mathrm{C}_{39} \mathrm{H}_{35} \mathrm{Cl}_{2} \mathrm{FN}_{6} \mathrm{O}_{4} \mathrm{~S}: \mathrm{C}, 60.54$; H, 4.58; N, 10.86. Found: C, 60.32; H, 4.28; N, 10.47.

2-Benzyl-5-((3aR,5S,6S,6aR)-6-((1-(4-chlorophenyl)1H-1,2,3-triazol-4-yl)methoxy)-2,2-dimethyltetrahydrofuro[2,3- $d][1,3]$ dioxol-5-yl)-3-(4-fluorophenyl)-6p-tolyl-3,3a,5,6-tetrahydro- $2 \mathrm{H}$-pyrazolo $[3,4-d]$ thiazole (10d). This was obtained by reacting compound $9 \mathrm{a}(1 \mathrm{~g})$ and benzylhydrazine $(0.65 \mathrm{~g})$ as described in the typical procedure and isolated as a brown solid, yield 66\%. m.p. $288-291^{\circ} \mathrm{C} ;{ }^{1} \mathrm{H}$ NMR $\left(300 \mathrm{MHz}, \mathrm{DMSO}-d_{6}\right) \delta 8.21(\mathrm{~d}, J=$ $8.7 \mathrm{~Hz}, 2 \mathrm{H}, \mathrm{Ar}-\mathrm{H}), 8.09$ (s, $1 \mathrm{H}, \mathrm{Ar}-\mathrm{H}), 7.55$ (d, $J=9.2 \mathrm{~Hz}$, $2 \mathrm{H}, \mathrm{Ar}-\mathrm{H}$ ), 7.42 (s, $1 \mathrm{H}, \mathrm{CH}-\mathrm{S}), 7.29$ (d, $J=8.33 \mathrm{~Hz}, 2 \mathrm{H}$, Ar-H), 7.10-7.08 (m, 5H, Ar-H), 7.05 (d, J=8.3 Hz, 2H, Ar-H), 6.91-6.87 (m, 4H, Ar-H), $5.92\left(\mathrm{~s}, 2 \mathrm{H}, \mathrm{CH}_{2} \mathrm{Ph}\right), 5.76$ $\left(\mathrm{d}, J=3.6 \mathrm{~Hz}, 1 \mathrm{H}, \mathrm{C}_{1} \mathrm{H}\right), 5.62(\mathrm{~d}, J=2.2 \mathrm{~Hz}, 1 \mathrm{H}, \mathrm{S}-\mathrm{CH})$, $5.25(\mathrm{~d}, J=2.2 \mathrm{~Hz}, 1 \mathrm{H}, \mathrm{CH}-\mathrm{N}), 4.66(\mathrm{t}, J=3.9 \mathrm{~Hz}, 1 \mathrm{H}$, $\left.\mathrm{C}_{2} \mathrm{H}\right), 4.54\left(\mathrm{~s}, 2 \mathrm{H}, \mathrm{OCH}_{2}\right), 3.96-3.94\left(\mathrm{~m}, 1 \mathrm{H}, \mathrm{C}_{4} \mathrm{H}\right), 3.26$ (dd, $\left.J_{1}=9.1 \mathrm{~Hz}, J_{2}=4.2 \mathrm{~Hz}, 1 \mathrm{H}, \mathrm{C}_{3} \mathrm{H}\right), 2.3\left(\mathrm{~s}, 3 \mathrm{H}, \mathrm{CH}_{3}\right)$, $1.53\left(\mathrm{~s}, 3 \mathrm{H}, \mathrm{CH}_{3}\right), 1.36\left(\mathrm{~m}, 3 \mathrm{H}, \mathrm{CH}_{3}\right) ;{ }^{13} \mathrm{C}$ NMR $(75 \mathrm{MHz}$, DMSO- $\left.d_{6}\right) \delta 171.6,151.6,143.2,137.4,133.6,132.3,131.2$, $129.3,125.3,128.4,127.9,124.8,122.9,119.2,111.2,103.8$, 81.2, 78.1, 74.1, 65.9, 58.1, 51.4, 26.1, 21.3, 16.1; MS: $\mathrm{m} / \mathrm{z}$ $\left(\mathrm{M}^{+}+\mathrm{H}\right)$ 753. Anal. Calcd for $\mathrm{C}_{40} \mathrm{H}_{38} \mathrm{ClFN}_{6} \mathrm{O}_{4} \mathrm{~S}: \mathrm{C}, 63.78$; H, 5.08; N, 11.16. Found: C, 63.52; H, 4.96; N, 10.97 .

5-((3aR,5S,6S,6aR)-6-((1-(4-Chlorophenyl)-1H-1,2,3triazol-4-yl)methoxy)-2,2-dimethyltetrahydrofuro[2,3- $d][1,3]$ dioxol-5-yl)-3-(4-fluorophenyl)-2-isopropyl-6-p-tolyl-3,3a,5,6-tetrahydro- $2 H$-pyrazolo $[3,4-d]$ thiazole (10e). This was obtained by reacting compound $9 \mathbf{a}(1 \mathrm{~g})$ and isopropylhydrazine $(0.35 \mathrm{~g})$ as described in the typical procedure and isolated as a yellow solid, yield 76\%, m.p. $248-251{ }^{\circ} \mathrm{C} ;{ }^{1} \mathrm{H}$ NMR (300 MHz, DMSO- $\left.d_{6}\right) \delta$ $8.19(\mathrm{~d}, J=8.7 \mathrm{~Hz}, 2 \mathrm{H}, \mathrm{Ar}-\mathrm{H}), 8.06(\mathrm{~s}, 1 \mathrm{H}, \mathrm{Ar}-\mathrm{H}), 7.55$ (d, $J=9.2 \mathrm{~Hz}, 2 \mathrm{H}, \mathrm{Ar}-\mathrm{H}), 7.42$ (s, 1H, CH-S) 7.29 (d, $J=8.33$ $\mathrm{Hz}, 2 \mathrm{H}, \mathrm{Ar}-\mathrm{H}$ ), 7.05 (d, $J=8.3 \mathrm{~Hz}, 2 \mathrm{H}, \mathrm{Ar}-\mathrm{H}), 6.91-6.87$ $(\mathrm{m}, 4 \mathrm{H}, \mathrm{Ar}-\mathrm{H}), 5.76\left(\mathrm{~d}, J=3.6 \mathrm{~Hz}, 1 \mathrm{H}, \mathrm{C}_{1} \mathrm{H}\right), 5.62(\mathrm{~d}, J=$ $2.2 \mathrm{~Hz}, 1 \mathrm{H}, \mathrm{S}-\mathrm{CH}), 5.25(\mathrm{~d}, J=2.2 \mathrm{~Hz}, 1 \mathrm{H}, \mathrm{CH}-\mathrm{N}), 4.66(\mathrm{t}$, $\left.J=3.9 \mathrm{~Hz}, 1 \mathrm{H}, \mathrm{C}_{2} \mathrm{H}\right), 4.54\left(\mathrm{~s}, 2 \mathrm{H}, \mathrm{OCH}_{2}\right), 3.96-3.94(\mathrm{~m}$, $\left.1 \mathrm{H}, \mathrm{C}_{4} \mathrm{H}\right), 3.26\left(\mathrm{dd}, J_{1}=9.1 \mathrm{~Hz}, J_{2}=4.2 \mathrm{~Hz}, 1 \mathrm{H}, \mathrm{C}_{3} \mathrm{H}\right), 2.80$ $2.78(\mathrm{~m}, 1 \mathrm{H}, \mathrm{CH}), 2.3\left(\mathrm{~s}, 3 \mathrm{H}_{1} \mathrm{CH}_{3}\right), 1.53\left(\mathrm{~s}, 3 \mathrm{H}, \mathrm{CH}_{3}\right), 1.36$ $\left(\mathrm{m}, 3 \mathrm{H}, \mathrm{CH}_{3}\right), 0.96\left(\mathrm{~d}, J=6.3 \mathrm{~Hz}, 6 \mathrm{H}, 2 \times \mathrm{CH}_{3}\right) ;{ }^{13} \mathrm{C} \mathrm{NMR}$ $\left(75 \mathrm{MHz}, \mathrm{DMSO}-d_{6}\right) \delta 171.6,151.6,143.2,137.4,133.6$, $132.3,131.2,129.3,128.4,127.9,124.8,122.9,119.2,111.2$, $103.8,81.2,78.1,74.1,65.9,58.1,51.4,50.6,26.1$, 21.3, 16.1; MS: $m / z\left(\mathrm{M}^{+}+\mathrm{H}\right)$ 705. Anal. Calcd for

Srinivaset al.: Synthesis, Nematicidal and Antifungal Properties ... 
$\mathrm{C}_{36} \mathrm{H}_{38} \mathrm{ClFN}_{6} \mathrm{O}_{4} \mathrm{~S}: \mathrm{C}, 61.31 ; \mathrm{H}, 5.43 ; \mathrm{N}, 11.92$. Found: C, $60.98 ; \mathrm{H}, 5.26 ; \mathrm{N}, 11.77$.

5-((3aR,5S,6S,6aR)-6-((1-(4-Chlorophenyl)-1H-1,2,3triazol-4-yl)methoxy)-2,2-dimethyltetrahydrofuro[2,3-d][1,3] dioxol-5-yl)-3-(4-fluorophenyl)-2-methyl-6-p-tolyl-3,3a,5,6-tetrahydro- $2 H$-pyrazolo $[3,4-d]$ thiazole $(\mathbf{1 0} \mathrm{f})$. This was obtained by reacting compound $9 \mathrm{a}$ $(1 \mathrm{~g})$ and methylhydrazine $(0.25 \mathrm{~g})$ as described in the typical procedure and isolated as a yellow solid, yield $66 \%$, m.p. $228-231^{\circ} \mathrm{C} ;{ }^{1} \mathrm{H}$ NMR $\left(300 \mathrm{MHz}\right.$, DMSO- $\left.d_{6}\right) \delta 8.19$ (d, $J=8.7 \mathrm{~Hz}, 2 \mathrm{H}, \mathrm{Ar}-\mathrm{H}), 8.06(\mathrm{~s}, 1 \mathrm{H}, \mathrm{Ar}-\mathrm{H}), 7.55$ (d, $J=9.2$ $\mathrm{Hz}, 2 \mathrm{H}, \mathrm{Ar}-\mathrm{H}$ ), 7.42 (s, 1H, CH-S), 7.29 (d, $J=8.33 \mathrm{~Hz}, 2 \mathrm{H}$, Ar-H), $7.05(\mathrm{~d}, J=8.3 \mathrm{~Hz}, 2 \mathrm{H}, \mathrm{Ar}-\mathrm{H}), 6.91-6.87(\mathrm{~m}, 4 \mathrm{H}$, Ar- $\mathrm{H}), 5.76\left(\mathrm{~d}, J=3.6 \mathrm{~Hz}, 1 \mathrm{H}, \mathrm{C}_{1} \mathrm{H}\right), 5.62(\mathrm{~d}, J=2.2 \mathrm{~Hz}$, $1 \mathrm{H}, \mathrm{S}-\mathrm{CH}), 5.25(\mathrm{~d}, J=2.2 \mathrm{~Hz}, 1 \mathrm{H}, \mathrm{CH}-\mathrm{N}), 4.66(\mathrm{t}, J=3.9$ $\left.\mathrm{Hz}, 1 \mathrm{H}, \mathrm{C}_{2} \mathrm{H}\right), 4.54\left(\mathrm{~s}, 2 \mathrm{H}, \mathrm{OCH}_{2}\right), 3.96-3.94\left(\mathrm{~m}, 1 \mathrm{H}, \mathrm{C}_{4} \mathrm{H}\right)$, $3.71\left(\mathrm{~s}, 3 \mathrm{H}, \mathrm{N}-\mathrm{CH}_{3}\right), 3.26\left(\mathrm{dd}, J_{1}=9.1 \mathrm{~Hz}, J_{2}=4.2 \mathrm{~Hz}, 1 \mathrm{H}\right.$, $\left.\mathrm{C}_{3} \mathrm{H}\right), 2.3\left(\mathrm{~s}, 3 \mathrm{H}, \mathrm{CH}_{3}\right), 1.53\left(\mathrm{~s}, 3 \mathrm{H}, \mathrm{CH}_{3}\right), 1.36(\mathrm{~m}, 3 \mathrm{H}$, $\left.\mathrm{CH}_{3}\right) ;{ }^{13} \mathrm{C}$ NMR $\left(75 \mathrm{MHz}\right.$, DMSO- $\left.d_{6}\right) \delta 171.6,151.6,143.2$, $137.4,133.6,132.3,131.2,129.3,128.4,127.9,124.8$, 122.9, 119.2, 111.2, 103.8, 81.2, 78.1, 74.1, 65.9, 58.1, 51.4, 39.3, 26.1, 16.1; MS: $m / z\left(\mathrm{M}^{+}+\mathrm{Na}+\right)$ 689. Anal. Calcd for $\mathrm{C}_{34} \mathrm{H}_{34} \mathrm{ClFN}_{6} \mathrm{O}_{4} \mathrm{~S}: \mathrm{C}, 60.30 ; \mathrm{H}, 5.06 ; \mathrm{N}, 12.42$. Found: $\mathrm{C}$, 60.22; H, 4.96: N, 12.22 .

6-(4-Chlorophenyl)-5-((3aR,5S,6S,6aR)-6-((1-(4-chlorophenyl)-1 $H$-1,2,3-triazol-4-yl)methoxy)-2,2-dimethyltetrahydrofuro $[2,3-d][1,3]$ dioxol-5-yl)-3-(4-fluorophenyl)-2-phenyl-3,3a,5,6-tetrahydro- $2 \mathrm{H}$-pyrazolo[3,4- $d]$ thiazole $(10 \mathrm{~g})$. This was obtained by reacting compound $9 \mathbf{b}(1 \mathrm{~g})$ and phenylhydrazine $(0.25 \mathrm{~g})$ as described in the typical procedure and isolated as a brown solid, yield $65 \%$, m.p. $249-251^{\circ} \mathrm{C} ;{ }^{1} \mathrm{H}$ NMR $(300 \mathrm{MHz}$, DMSO- $\left.d_{6}\right) \delta 8.16(\mathrm{~d}, J=8.7 \mathrm{~Hz}, 2 \mathrm{H}, \mathrm{Ar}-\mathrm{H}), 8.02(\mathrm{~s}, 1 \mathrm{H}$, Ar-H), 7.49 (d, J = 9.2 Hz, 2H, Ar-H), 7.36 (s, 1H, CH-S), $7.22(\mathrm{~d}, J=8.33 \mathrm{~Hz}, 2 \mathrm{H}, \mathrm{Ar}-\mathrm{H}), 7.13-7.11(\mathrm{~m}, 5 \mathrm{H}, \mathrm{Ar}-\mathrm{H})$, $7.02(\mathrm{~d}, J=8.3 \mathrm{~Hz}, 2 \mathrm{H}, \mathrm{Ar}-\mathrm{H}), 6.81-6.77(\mathrm{~m}, 4 \mathrm{H}, \mathrm{Ar}-\mathrm{H})$, $5.66\left(\mathrm{~d}, J=3.6 \mathrm{~Hz}, 1 \mathrm{H}, \mathrm{C}_{1} \mathrm{H}\right), 5.58(\mathrm{~d}, J=2.2 \mathrm{~Hz}, 1 \mathrm{H}$, $\mathrm{S}-\mathrm{CH}), 5.21(\mathrm{~d}, J=2.2 \mathrm{~Hz}, 1 \mathrm{H}, \mathrm{CH}-\mathrm{N}), 4.62(\mathrm{t}, J=3.9 \mathrm{~Hz}$, $\left.1 \mathrm{H}, \mathrm{C}_{2} \mathrm{H}\right), 4.51\left(\mathrm{~s}, 2 \mathrm{H}, \mathrm{OCH}_{2}\right), 3.94-3.91\left(\mathrm{~m}, 1 \mathrm{H}, \mathrm{C}_{4} \mathrm{H}\right)$, $3.26\left(\mathrm{dd}, J_{1}=9.1 \mathrm{~Hz}, J_{2}=4.2 \mathrm{~Hz}, 1 \mathrm{H}, \mathrm{C}_{3} \mathrm{H}\right), 1.53(\mathrm{~s}, 3 \mathrm{H}$, $\left.\mathrm{CH}_{3}\right), 1.36\left(\mathrm{~m}, 3 \mathrm{H}, \mathrm{CH}_{3}\right) ;{ }^{13} \mathrm{C}$ NMR $\left(75 \mathrm{MHz}\right.$, DMSO- $\left.d_{6}\right) \delta$ 174.6, 143.0, 141.4, 137.4, 133.6, 132.3, 131.2, 129.3, 125.3, $128.4,127.9,126.1,124.8,122.9,119.2,111.2,103.8,81.2$, 78.1, 74.1, 65.9, 51.4, 26.1, 16.1; MS: $m / z\left(\mathrm{M}^{+}+\mathrm{H}\right) 759$. Anal. Calcd for $\mathrm{C}_{39} \mathrm{H}_{36} \mathrm{ClFN}_{6} \mathrm{O}_{4} \mathrm{~S}: \mathrm{C}, 60.08 ; \mathrm{H}, 4.38 ; \mathrm{N}$, 11.07. Found: C, $60.02 ; \mathrm{H}, 4.11 ; \mathrm{N}, 10.95$.

6-(4-Chlorophenyl)-5-((3aR,5S,6S,6aR)-6-((1-(4-chlorophenyl)-1H-1,2,3-triazol-4-yl)methoxy)-2,2-dimethyltetrahydrofuro[2,3-d][1,3] dioxol-5-yl)-3-(4-fluorophenyl)-2-(4-methoxyphenyl)-3,3a,5,6-tetrahydro- $2 H$-pyrazolo[3,4- $d$ ] thiazole $(\mathbf{1 0 h})$. This was obtained by reacting compound $9 \mathbf{b}(1 \mathrm{~g})$ and $p$-methoxyphenylhydrazine $(0.35 \mathrm{~g})$ as described in the typical proce- dure and isolated as a yellow solid, yield 68\%, m.p. 274-276 ${ }^{\circ} \mathrm{C} ;{ }^{1} \mathrm{H}$ NMR $\left(300 \mathrm{MHz}\right.$, DMSO- $\left.d_{6}\right) \delta 8.18(\mathrm{~d}, J=8.7 \mathrm{~Hz}$, $2 \mathrm{H}, \operatorname{Ar}-\mathrm{H}), 8.04(\mathrm{~s}, 1 \mathrm{H}, \mathrm{Ar}-\mathrm{H}), 7.51(\mathrm{~d}, J=9.2 \mathrm{~Hz}, 2 \mathrm{H}$, Ar-H), 7.40 (s, 1H, CH-S), 7.29 (d, $J=8.33 \mathrm{~Hz}, 2 \mathrm{H}, \mathrm{Ar}-\mathrm{H})$, $7.12-7.10(\mathrm{~m}, 4 \mathrm{H}, \mathrm{Ar}-\mathrm{H}), 7.05(\mathrm{~d}, J=8.3 \mathrm{~Hz}, 2 \mathrm{H}, \mathrm{Ar}-\mathrm{H})$, 6.91-6.87 (m, 4H, Ar-H), $5.76\left(\mathrm{~d}, J=3.6 \mathrm{~Hz}, 1 \mathrm{H}, \mathrm{C}_{1} \mathrm{H}\right)$, $5.62(\mathrm{~d}, J=2.2 \mathrm{~Hz}, 1 \mathrm{H}, \mathrm{S}-\mathrm{CH}), 5.25(\mathrm{~d}, J=2.2 \mathrm{~Hz}, 1 \mathrm{H}$, CH-N), $4.66\left(\mathrm{t}, J=3.9 \mathrm{~Hz}, 1 \mathrm{H}, \mathrm{C}_{2} \mathrm{H}\right), 4.54\left(\mathrm{~s}, 2 \mathrm{H}, \mathrm{OCH}_{2}\right)$, 3.96-3.94 (m, $\left.1 \mathrm{H}, \mathrm{C}_{4} \mathrm{H}\right), 3.91(\mathrm{~s}, 3 \mathrm{H}, \mathrm{OMe}), 3.26\left(\mathrm{dd}, J_{1}=\right.$ $\left.9.1 \mathrm{~Hz}, J_{2}=4.2 \mathrm{~Hz}, 1 \mathrm{H}, \mathrm{C}_{3} \mathrm{H}\right), 1.53\left(\mathrm{~s}, 3 \mathrm{H}, \mathrm{CH}_{3}\right), 1.36(\mathrm{~m}$, $\left.3 \mathrm{H}, \mathrm{CH}_{3}\right) ;{ }^{13} \mathrm{C}$ NMR $\left(75 \mathrm{MHz}\right.$, DMSO- $\left.d_{6}\right) \delta 172.6,151.7$, $143.2,141.4,137.4,133.6,132.3,131.2,129.3,126.1,125.3$, $128.4,127.9,124.8,122.9,119.2,111.2,103.8,81.2,78.1$, 74.1, 65.9, 55.8, 51.4, 26.1, 16.1; MS: $m / z\left(\mathrm{M}^{+}+\mathrm{H}\right) 789$. Anal. Calcd for $\mathrm{C}_{39} \mathrm{H}_{35} \mathrm{Cl}_{2} \mathrm{FN}_{6} \mathrm{O}_{5} \mathrm{~S}: \mathrm{C}, 59.32 ; \mathrm{H}, 4.47 ; \mathrm{N}$, 10.64. Found: C, 58.92; H, 4.18; N, 10.47 .

2,6-Bis(4-chlorophenyl)-5-((3aR,5S,6S,6aR)-6-((1-(4chlorophenyl)-1H-1,2,3-triazol-4-yl)methoxy)-2,2-dimethyltetrahydrofuro [2,3-d][1,3] dioxol-5-yl)-3-(4-fluorophenyl)-3,3a,5,6-tetrahydro- $2 \mathrm{H}$-pyrazolo $[3,4-d]$ thiazole (10i). This was obtained by reacting compound $\mathbf{9 b}$ $(1 \mathrm{~g})$ and $p$-chlorophenylhydrazine $(0.45 \mathrm{~g})$ as described in the typical procedure and isolated as a brown solid, yield 76\%, m.p. $268-271{ }^{\circ} \mathrm{C} ;{ }^{1} \mathrm{H}$ NMR (300 MHz, DMSO- $\left.d_{6}\right) \delta$ $8.23(\mathrm{~d}, J=8.7 \mathrm{~Hz}, 2 \mathrm{H}, \mathrm{Ar}-\mathrm{H}), 8.11(\mathrm{~s}, 1 \mathrm{H}, \mathrm{Ar}-\mathrm{H}), 7.58$ (d, $J=9.2 \mathrm{~Hz}, 2 \mathrm{H}, \mathrm{Ar}-\mathrm{H}), 7.46$ (s, 1H, CH-S), 7.29 (d, $J=8.33$ $\mathrm{Hz}, 2 \mathrm{H}, \mathrm{Ar}-\mathrm{H}), 7.11-7.09$ (m, 4H, Ar-H), 7.07 (d, $J=8.3$ $\mathrm{Hz}, 2 \mathrm{H}, \mathrm{Ar}-\mathrm{H}), 6.91-6.87$ (m, 4H, Ar-H), 5.76 (d, $J=3.6$ $\left.\mathrm{Hz}, 1 \mathrm{H}, \mathrm{C}_{1} \mathrm{H}\right), 5.62(\mathrm{~d}, J=2.2 \mathrm{~Hz}, 1 \mathrm{H}, \mathrm{S}-\mathrm{CH}), 5.25(\mathrm{~d}, J=$ $2.2 \mathrm{~Hz}, 1 \mathrm{H}, \mathrm{CH}-\mathrm{N}), 4.66\left(\mathrm{t}, J=3.9 \mathrm{~Hz}, 1 \mathrm{H}, \mathrm{C}_{2} \mathrm{H}\right), 4.54(\mathrm{~s}$, $\left.2 \mathrm{H}, \mathrm{OCH}_{2}\right), 3.96-3.94\left(\mathrm{~m}, 1 \mathrm{H}, \mathrm{C}_{4} \mathrm{H}\right), 3.26\left(\mathrm{dd}, J_{1}=9.1 \mathrm{~Hz}\right.$, $\left.J_{2}=4.2 \mathrm{~Hz}, 1 \mathrm{H}, \mathrm{C}_{3} \mathrm{H}\right), 1.53\left(\mathrm{~s}, 3 \mathrm{H}, \mathrm{CH}_{3}\right), 1.36\left(\mathrm{~m}, 3 \mathrm{H}, \mathrm{CH}_{3}\right)$; ${ }^{13} \mathrm{C}$ NMR $(75 \mathrm{MHz}$, DMSO- $d$ ) $\delta 172.6,151.7,143.2,137.4$, $133.6,132.3,131.2,129.3,126.3,125.3,128.4,127.9,124.8$, $122.9,119.2,111.2,103.8,81.2,78.1,74.1,65.9,51.4$, 26.1, 16.1; MS: $m / z\left(\mathrm{M}^{+}+\mathrm{Na}\right)$ 815. Anal. Calcd for $\mathrm{C}_{38} \mathrm{H}_{32} \mathrm{Cl}_{3} \mathrm{FN}_{6} \mathrm{O}_{4} \mathrm{~S}: \mathrm{C}, 57.47 ; \mathrm{H}, 4.08 ; \mathrm{N}, 10.56$. Found: C, $57.22 ; \mathrm{H}, 3.95 ; \mathrm{N}, 10.32$.

2-Benzyl-6-(4-chlorophenyl)-5-((3aR,5S,6S,6aR)-6-((1(4-chlorophenyl)-1 H-1,2,3-triazol-4-yl)methoxy)-2,2dimethyltetrahydrofuro $[2,3-d][1,3]$ dioxol-5-yl) -3-(4-fluorophenyl)-3,3a,5,6-tetrahydro- $2 \mathrm{H}$-pyrazolo[3,4- $\boldsymbol{d}]$ thiazole $(\mathbf{1 0 j})$. This was obtained by reacting compound $9 \mathrm{~b}(1 \mathrm{~g})$ and benzylhydrazine $(0.65 \mathrm{~g})$ as described in the typical procedure and isolated as a brown solid, yield $71 \%$, m.p. $258-261{ }^{\circ} \mathrm{C} ;{ }^{1} \mathrm{H}$ NMR $(300 \mathrm{MHz}$, DMSO- $\left.d_{6}\right) \delta 8.25(\mathrm{~d}, J=8.7 \mathrm{~Hz}, 2 \mathrm{H}, \mathrm{Ar}-\mathrm{H}), 8.11(\mathrm{~s}, 1 \mathrm{H}$, Ar-H), 7.59 (d, J = 9.2 Hz, 2H, Ar-H), 7.46 (s, 1H, CH-S), $7.32(\mathrm{~d}, J=8.33 \mathrm{~Hz}, 2 \mathrm{H}, \mathrm{Ar}-\mathrm{H}), 7.12-7.10(\mathrm{~m}, 5 \mathrm{H}, \mathrm{Ar}-\mathrm{H})$, $7.08(\mathrm{~d}, J=8.3 \mathrm{~Hz}, 2 \mathrm{H}, \mathrm{Ar}-\mathrm{H}), 6.96-6.90(\mathrm{~m}, 4 \mathrm{H}, \mathrm{Ar}-\mathrm{H})$, $5.96\left(\mathrm{~s}, 2 \mathrm{H}, \mathrm{CH}_{2} \mathrm{Ph}\right), 5.77\left(\mathrm{~d}, J=3.6 \mathrm{~Hz}, 1 \mathrm{H}, \mathrm{C}_{1} \mathrm{H}\right), 5.65(\mathrm{~d}$, $J=2.2 \mathrm{~Hz}, 1 \mathrm{H}, \mathrm{S}-\mathrm{CH}), 5.28(\mathrm{~d}, J=2.2 \mathrm{~Hz}, 1 \mathrm{H}, \mathrm{CH}-\mathrm{N}), 4.66$ $\left(\mathrm{t}, J=3.9 \mathrm{~Hz}, 1 \mathrm{H}, \mathrm{C}_{2} \mathrm{H}\right), 4.54\left(\mathrm{~s}, 2 \mathrm{H}, \mathrm{OCH}_{2}\right), 3.96-3.94(\mathrm{~m}$, $\left.1 \mathrm{H}, \mathrm{C}_{4} \mathrm{H}\right), 3.26\left(\mathrm{dd}, J_{1}=9.1 \mathrm{~Hz}, J_{2}=4.2 \mathrm{~Hz}, 1 \mathrm{H}, \mathrm{C}_{3} \mathrm{H}\right), 1.53$ 
(s, $\left.3 \mathrm{H}, \mathrm{CH}_{3}\right), 1.36\left(\mathrm{~m}, 3 \mathrm{H}, \mathrm{CH}_{3}\right) ;{ }^{13} \mathrm{C}$ NMR $(75 \mathrm{MHz}$, DMSO- $\left.d_{6}\right) \delta 171.6,151.6,143.2,137.4,133.6,132.3,131.2$, $129.3,125.3,128.4,127.9,124.8,122.9,119.2,111.2,103.8$, 81.2, 78.1, 74.1, 65.9, 58.1, 51.4, 26.1, 16.1; MS: $m / z\left(\mathrm{M}^{+}+\right.$ $\mathrm{H})$ 773. Anal. Calcd for $\mathrm{C}_{39} \mathrm{H}_{35} \mathrm{Cl}_{2} \mathrm{FNO}_{6} \mathrm{~S}$ : $\mathrm{C}, 60.54 ; \mathrm{H}$, 4.56; N, 10.86. Found: C, 60.34; H, 4.36; N, 10.37 .

6-(4-Chlorophenyl)-5-((3aR,5S,6S,6aR)-6-((1-(4-chlorophenyl)-1H-1,2,3-triazol-4-yl)methoxy)-2,2-dimethyltetrahydrofuro[2,3-d][1,3] dioxol-5-yl)-3-(4-fluorophenyl)-2-isopropyl-3,3a,5,6-tetrahydro- $2 \mathrm{H}$-pyrazolo $[3,4-d]$ thiazole $(10 \mathrm{k})$. This was obtained by reacting compound $9 \mathbf{b}(1 \mathrm{~g})$ and isopropylhydrazine $(0.35 \mathrm{~g})$ as described in the typical procedure and isolated as a yellow solid, yield $69 \%$, m.p. $238-239{ }^{\circ} \mathrm{C}$; ${ }^{1} \mathrm{H}$ NMR $(300 \mathrm{MHz}$, DMSO- $\left.d_{6}\right) \delta 8.32(\mathrm{~d}, J=8.7 \mathrm{~Hz}, 2 \mathrm{H}$, Ar- $\mathrm{H}), 8.16$ (s,1H,Ar-H), $7.65(\mathrm{~d}, J=9.2 \mathrm{~Hz}, 2 \mathrm{H}, \mathrm{Ar}-\mathrm{H}), 7.52(\mathrm{~s}, 1 \mathrm{H}$, CH-S), 7.39 (d, $J=8.33 \mathrm{~Hz}, 2 \mathrm{H}, \mathrm{Ar}-\mathrm{H}), 7.09$ (d, $J=8.3 \mathrm{~Hz}$, $2 \mathrm{H}, \mathrm{Ar}-\mathrm{H}), 6.95-6.92(\mathrm{~m}, 4 \mathrm{H}, \mathrm{Ar}-\mathrm{H}), 5.76(\mathrm{~d}, J=3.6 \mathrm{~Hz}$, $\left.1 \mathrm{H}, \mathrm{C}_{1} \mathrm{H}\right), 5.62(\mathrm{~d}, J=2.2 \mathrm{~Hz}, 1 \mathrm{H}, \mathrm{S}-\mathrm{CH}), 5.25(\mathrm{~d}, J=2.2$ $\mathrm{Hz}, 1 \mathrm{H}, \mathrm{CH}-\mathrm{N}), 4.66\left(\mathrm{t}, J=3.9 \mathrm{~Hz}, 1 \mathrm{H}, \mathrm{C}_{2} \mathrm{H}\right), 4.54(\mathrm{~s}, 2 \mathrm{H}$, $\left.\mathrm{OCH}_{2}\right), 3.96-3.94\left(\mathrm{~m}, 1 \mathrm{H}, \mathrm{C}_{4} \mathrm{H}\right), 3.26\left(\mathrm{dd}, J_{1}=9.1 \mathrm{~Hz}, J_{2}=\right.$ $\left.4.2 \mathrm{~Hz}, 1 \mathrm{H}, \mathrm{C}_{3} \mathrm{H}\right), 2.80-2.78(\mathrm{~m}, 1 \mathrm{H}, \mathrm{CH}), 1.53(\mathrm{~s}, 3 \mathrm{H}$, $\left.\mathrm{CH}_{3}\right), 1.36\left(\mathrm{~m}, 3 \mathrm{H}, \mathrm{CH}_{3}\right), 0.96\left(\mathrm{~d}, J=6.3 \mathrm{~Hz}, 6 \mathrm{H}, 2 \times \mathrm{CH}_{3}\right)$; ${ }^{13} \mathrm{C}$ NMR $\left(75 \mathrm{MHz}\right.$, DMSO- $\left.d_{6}\right) \delta 171.6,151.6,143.2,137.4$, $133.6,132.3,131.2,129.3,128.4,127.9,124.8,122.9,119.2$, $111.2,103.8,81.2,78.1,74.1,65.9,58.1,51.4,50.6$, 26.1, 16.1; MS: $m / z\left(\mathrm{M}^{+}+\mathrm{H}\right)$ 725. Anal. Calcd for $\mathrm{C}_{35} \mathrm{H}_{35} \mathrm{Cl}_{2} \mathrm{FN}_{6} \mathrm{O}_{4} \mathrm{~S}: \mathrm{C}, 57.93 ; \mathrm{H}, 4.86 ; \mathrm{N}, 11.58$. Found: C, $57.72 ; \mathrm{H}, 4.66 ; \mathrm{N}, 11.46$.

6-(4-Chlorophenyl)-5-((3aR,5S,6S,6aR)-6-((1-(4-chlorophenyl)-1H-1,2,3-triazol-4-yl)methoxy)-2,2-dimethyltetrahydrofuro [2,3-d][1,3] dioxol-5-yl)-3-(4-fluorophenyl)-2-methyl-3,3a,5,6-tetrahydro-2H-pyrazolo[3,4- $\boldsymbol{d}]$ thiazole (101). This was obtained by reacting compound $9 \mathbf{b}(1 \mathrm{~g})$ and methylhydrazine $(0.25 \mathrm{~g})$ as described in the typical procedure and isolated as a yellow solid, yield 76\%, m.p. $241-243{ }^{\circ} \mathrm{C} ;{ }^{1} \mathrm{H}$ NMR $(300 \mathrm{MHz}$, DMSO-d $d_{6} \delta 8.29(\mathrm{~d}, J=8.7 \mathrm{~Hz}, 2 \mathrm{H}, \mathrm{Ar}-\mathrm{H}), 8.16(\mathrm{~s}, 1 \mathrm{H}$, Ar-H), 7.59 (d, $J=9.2 \mathrm{~Hz}, 2 \mathrm{H}, \mathrm{Ar}-\mathrm{H}), 7.48$ (s, 1H, CH-S), $7.39(\mathrm{~d}, J=8.33 \mathrm{~Hz}, 2 \mathrm{H}, \mathrm{Ar}-\mathrm{H}), 7.09(\mathrm{~d}, J=8.3 \mathrm{~Hz}, 2 \mathrm{H}, \mathrm{Ar}-$ $\mathrm{H}), 6.91-6.88(\mathrm{~m}, 4 \mathrm{H}, \mathrm{Ar}-\mathrm{H}), 5.76\left(\mathrm{~d}, J=3.6 \mathrm{~Hz}, 1 \mathrm{H}, \mathrm{C}_{1} \mathrm{H}\right)$, $5.62(\mathrm{~d}, J=2.2 \mathrm{~Hz}, 1 \mathrm{H}, \mathrm{S}-\mathrm{CH}), 5.25(\mathrm{~d}, J=2.2 \mathrm{~Hz}, 1 \mathrm{H}, \mathrm{CH}-$ $\mathrm{N}), 4.66\left(\mathrm{t}, J=3.9 \mathrm{~Hz}, 1 \mathrm{H}, \mathrm{C}_{2} \mathrm{H}\right), 4.54\left(\mathrm{~s}, 2 \mathrm{H}, \mathrm{OCH}_{2}\right), 3.96-$ $3.94\left(\mathrm{~m}, 1 \mathrm{H}, \mathrm{C}_{4} \mathrm{H}\right), 3.71\left(\mathrm{~s}, 3 \mathrm{H}, \mathrm{N}-\mathrm{CH}_{3}\right), 3.26\left(\mathrm{dd}, J_{1}=9.1\right.$ $\left.\mathrm{Hz}, J_{2}=4.2 \mathrm{~Hz}, 1 \mathrm{H}, \mathrm{C}_{3} \mathrm{H}\right), 1.53\left(\mathrm{~s}, 3 \mathrm{H}, \mathrm{CH}_{3}\right), 1.36(\mathrm{~m}, 3 \mathrm{H}$, $\left.\mathrm{CH}_{3}\right) ;{ }^{13} \mathrm{C}$ NMR (75 MHz, DMSO- $\left.d_{6}\right) \delta 171.6,151.6,143.2$, 137.4, 133.6, 132.3, 131.2, 129.3, 128.4, 127.9, 124.8, 122.9, 119.2, 111.2, 103.8, 81.2, 78.1, 74.1, 65.9, 58.1, 51.4, 39.3, 16.1; MS: $m / z\left(\mathrm{M}^{+}+\mathrm{H}\right)$ 684. Anal. Calcd for $\mathrm{C}_{32} \mathrm{H}_{30} \mathrm{Cl}_{2} \mathrm{FN}_{6} \mathrm{O}_{4} \mathrm{~S}: \mathrm{C}, 56.14 ; \mathrm{H}, 4.42 ; \mathrm{N}, 12.28$. Found: C, 55.92; H, 4.26: N, 12.02 .

5-((3aR,5S,6S,6aR)-6-((1-(4-Chlorophenyl)-1H-1,2,3triazol-4-yl)methoxy)-2,2-dimethyltetrahydrofu- ro[2,3-d][1,3]dioxol-5-yl)-3-(4-fluorophenyl)-6-(4-nitrophenyl)-2-phenyl-3,3a,5,6-tetrahydro- $2 \mathrm{H}$-pyrazolo $[3,4-d]$ thiazole $(10 \mathrm{~m})$. This was obtained by reacting compound $9 \mathrm{c}(1 \mathrm{~g})$ and phenylhydrazine $(0.25 \mathrm{~g})$ as described in the typical procedure and isolated as a brown solid, yield $75 \%$, m.p. $241-243{ }^{\circ} \mathrm{C} ;{ }^{1} \mathrm{H}$ NMR $(300 \mathrm{MHz}$, DMSO- $\left.d_{6}\right) \delta 8.26(\mathrm{~d}, J=8.7 \mathrm{~Hz}, 2 \mathrm{H}, \mathrm{Ar}-\mathrm{H}), 8.02(\mathrm{~s}, 1 \mathrm{H}$, Ar-H), 7.49 (d, $J=9.2 \mathrm{~Hz}, 2 \mathrm{H}, \mathrm{Ar}-\mathrm{H}), 7.22(\mathrm{~d}, J=8.33 \mathrm{~Hz}$, 2H, Ar-H), 7.08-7.05 (m, 6H, Ar-H and CH-S), 7.02 (d, J $=8.3 \mathrm{~Hz}, 2 \mathrm{H}, \mathrm{Ar}-\mathrm{H}), 6.81-6.77(\mathrm{~m}, 4 \mathrm{H}, \mathrm{Ar}-\mathrm{H}), 5.66(\mathrm{~d}, J=$ $\left.3.6 \mathrm{~Hz}, 1 \mathrm{H}, \mathrm{C}_{1} \mathrm{H}\right), 5.58(\mathrm{~d}, J=2.2 \mathrm{~Hz}, 1 \mathrm{H}, \mathrm{S}-\mathrm{CH}), 5.21(\mathrm{~d}, J$ $=2.2 \mathrm{~Hz}, 1 \mathrm{H}, \mathrm{CH}-\mathrm{N}), 4.62\left(\mathrm{t}, J=3.9 \mathrm{~Hz}, 1 \mathrm{H}, \mathrm{C}_{2} \mathrm{H}\right), 4.51(\mathrm{~s}$, $\left.2 \mathrm{H}, \mathrm{OCH}_{2}\right), 3.94-3.91\left(\mathrm{~m}, 1 \mathrm{H}, \mathrm{C}_{4} \mathrm{H}\right), 3.26\left(\mathrm{dd}, J_{1}=9.1 \mathrm{~Hz}\right.$, $\left.J_{2}=4.2 \mathrm{~Hz}, 1 \mathrm{H}, \mathrm{C}_{3} \mathrm{H}\right), 1.53\left(\mathrm{~s}, 3 \mathrm{H}, \mathrm{CH}_{3}\right), 1.36\left(\mathrm{~m}, 3 \mathrm{H}, \mathrm{CH}_{3}\right)$; ${ }^{13} \mathrm{C}$ NMR $(75 \mathrm{MHz}$, DMSO- $d$ ) $\delta 174.6,143.0,141.4,137.4$, $136.2,133.6,132.3,131.2,129.3,128.4,127.9,126.1,124.8$, $122.9,119.2,111.2,103.8,81.2,78.1,74.1,65.9,51.4$, 26.1, 16.1; MS: $m / z\left(\mathrm{M}^{+}+\mathrm{H}\right)$ 770. Anal. Calcd for $\mathrm{C}_{38} \mathrm{H}_{33} \mathrm{ClFN}_{7} \mathrm{O}_{6} \mathrm{~S}: \mathrm{C}, 59.26 ; \mathrm{H}, 4.32 ; \mathrm{N}, 12.73$. Found: $\mathrm{C}$, 59.16; H, 4.10; N, 12.53 .

5-((3aR,5S,6S,6aR)-6-((1-(4-Chlorophenyl)-1H-1,2,3triazol-4-yl)methoxy)-2,2-dimethyltetrahydrofuro[2,3-d][1,3] dioxol-5-yl)-3-(4-fluorophenyl)-2-(4-methoxyphenyl)-6-(4-nitrophenyl)-3,3a,5,6-tetrahydro-2H-pyrazolo[3,4-d] thiazole (10n). This was obtained by reacting compound $9 \mathrm{c}(1 \mathrm{~g})$ and $p$-methoxyphenylhydrazine $(0.35 \mathrm{~g})$ as described in the typical procedure and isolated as a yellow solid, yield 76\%, m.p. 264-266 ${ }^{\circ} \mathrm{C} ;{ }^{1} \mathrm{H}$ NMR $\left(300 \mathrm{MHz}, \mathrm{DMSO}-d_{6}\right) \delta 8.28(\mathrm{~d}, J=8.7 \mathrm{~Hz}$, $2 \mathrm{H}, \mathrm{Ar}-\mathrm{H}), 8.04(\mathrm{~s}, 1 \mathrm{H}, \mathrm{Ar}-\mathrm{H}), 7.51(\mathrm{~d}, J=9.2 \mathrm{~Hz}, 2 \mathrm{H}, \mathrm{Ar}-$ $\mathrm{H}$ ), 7.40-7.35 (m, 5H, Ar-H and CH-S), 7.29 (d, J= 8.33 $\mathrm{Hz}, 2 \mathrm{H}, \mathrm{Ar}-\mathrm{H}), 7.05$ (d, $J=8.3 \mathrm{~Hz}, 2 \mathrm{H}, \mathrm{Ar}-\mathrm{H}), 6.91-6.87$ $(\mathrm{m}, 4 \mathrm{H}, \mathrm{Ar}-\mathrm{H}), 5.76\left(\mathrm{~d}, J=3.6 \mathrm{~Hz}, 1 \mathrm{H}, \mathrm{C}_{1} \mathrm{H}\right), 5.62(\mathrm{~d}, J=$ $2.2 \mathrm{~Hz}, 1 \mathrm{H}, \mathrm{S}-\mathrm{CH}), 5.25(\mathrm{~d}, J=2.2 \mathrm{~Hz}, 1 \mathrm{H}, \mathrm{CH}-\mathrm{N}), 4.66(\mathrm{t}$, $\left.J=3.9 \mathrm{~Hz}, 1 \mathrm{H}, \mathrm{C}_{2} \mathrm{H}\right), 4.54\left(\mathrm{~s}, 2 \mathrm{H}, \mathrm{OCH}_{2}\right), 3.96-3.94(\mathrm{~m}$, $\left.1 \mathrm{H}, \mathrm{C}_{4} \mathrm{H}\right), 3.91(\mathrm{~s}, 3 \mathrm{H}, \mathrm{OMe}), 3.26\left(\mathrm{dd}, J_{1}=9.1 \mathrm{~Hz}, J_{2}=4.2\right.$ $\left.\mathrm{Hz}, 1 \mathrm{H}, \mathrm{C}_{3} \mathrm{H}\right), 1.53\left(\mathrm{~s}, 3 \mathrm{H}, \mathrm{CH}_{3}\right), 1.36\left(\mathrm{~m}, 3 \mathrm{H}, \mathrm{CH}_{3}\right) ;{ }^{13} \mathrm{C}$ NMR $\left(75 \mathrm{MHz}\right.$, DMSO- $\left.d_{6}\right) \delta 172.6,151.7,143.2,141.4$, $137.4,133.6,132.3,131.2,129.3,126.1,125.3,128.4,127.9$, 124.8, 122.9, 119.2, 111.2, 103.8, 81.2, 78.1, 74.1, 65.9, 55.8, 51.4, 26.1, 16.1; MS: $m / z\left(\mathrm{M}^{+}+\mathrm{H}\right)$ 800. Anal. Calcd for $\mathrm{C}_{39} \mathrm{H}_{35} \mathrm{ClFN}_{7} \mathrm{O}_{7} \mathrm{~S}$ : C, 58.53; H, 4.41; N, 12.25. Found: C, $58.22 ; \mathrm{H}, 4.16 ; \mathrm{N}, 12.01$.

2-(4-Chlorophenyl)-5-((3aR,5S,6S,6aR)-6-((1-(4-chlorophenyl)-1H-1,2,3-triazol-4-yl)methoxy)-2,2-dimethyltetrahydrofuro [2,3-d] [1,3] dioxol-5-yl)-3-(4-fluorophenyl)-6-(4-nitrophenyl)-3,3a,5,6-tetrahydro-2H-pyrazolo[3,4- $d]$ thiazole $(10$ o). This was obtained by reacting compound $9 \mathrm{c}(1 \mathrm{~g})$ and $p$-chlorophenylhydrazine $(0.45 \mathrm{~g})$ as described in the typical procedure and isolated as a brown solid, yield $72 \%$, m.p. $285-287^{\circ} \mathrm{C} ;{ }^{1} \mathrm{H}$ NMR $(300 \mathrm{MHz}$, DMSO- $\left.d_{6}\right) \delta 8.23(\mathrm{~d}, J=8.7 \mathrm{~Hz}, 2 \mathrm{H}, \mathrm{Ar}-\mathrm{H}), 8.11(\mathrm{~s}, 1 \mathrm{H}, \mathrm{Ar}-\mathrm{H})$, $7.58(\mathrm{~d}, J=9.2 \mathrm{~Hz}, 2 \mathrm{H}, \mathrm{Ar}-\mathrm{H}), 7.40-7.35$ (m, 5H, Ar-H and CH-S), 7.29 (d, $J=8.33 \mathrm{~Hz}, 2 \mathrm{H}, \mathrm{Ar}-\mathrm{H}), 7.07$ (d, $J=8.3 \mathrm{~Hz}$,

Srinivaset al.: Synthesis, Nematicidal and Antifungal Properties ... 
2H, Ar-H), 6.91-6.87 (m, 4H, Ar-H), 5.76 (d, J=3.6 Hz, $1 \mathrm{H}$, $\left.\mathrm{C}_{1} \mathrm{H}\right), 5.62(\mathrm{~d}, J=2.2 \mathrm{~Hz}, 1 \mathrm{H}, \mathrm{S}-\mathrm{CH}), 5.25(\mathrm{~d}, J=2.2 \mathrm{~Hz}, 1 \mathrm{H}$, $\mathrm{CH}-\mathrm{N}), 4.66\left(\mathrm{t}, J=3.9 \mathrm{~Hz}, 1 \mathrm{H}, \mathrm{C}_{2} \mathrm{H}\right), 4.54\left(\mathrm{~s}, 2 \mathrm{H}, \mathrm{OCH}_{2}\right)$, 3.96-3.94 (m, 1H, C 4 H), $3.26\left(\mathrm{dd}, J_{1}=9.1 \mathrm{~Hz}, J_{2}=4.2 \mathrm{~Hz}\right.$, $\left.1 \mathrm{H}, \mathrm{C}_{3} \mathrm{H}\right), 1.53\left(\mathrm{~s}, 3 \mathrm{H}, \mathrm{CH}_{3}\right), 1.36\left(\mathrm{~m}, 3 \mathrm{H}, \mathrm{CH}_{3}\right) ;{ }^{13} \mathrm{C} \mathrm{NMR}$ $\left(75 \mathrm{MHz}, \mathrm{DMSO}-d_{6}\right) \delta 172.6,151.7,143.2,137.4,133.6$, $132.3,131.2,129.3,126.3,125.3,128.4,127.9,124.8,122.9$, 119.2, 111.2, 103.8, 81.2, 78.1, 74.1, 65.9, 51.4, 26.1, 16.1; MS: $m / z\left(\mathrm{M}^{+}+\mathrm{Na}\right)$ 815. Anal. Calcd for $\mathrm{C}_{38} \mathrm{H}_{32} \mathrm{Cl}_{2} \mathrm{FN}_{7} \mathrm{O}_{6} \mathrm{~S}: \mathrm{C}$, 56.72; H, 4.01; N, 12.81. Found: C, 56.42; H, 3.95; N, 12.72.

2-Benzyl-5-((3aR,5S,6S,6aR)-6-((1-(4-chlorophenyl)1H-1,2,3-triazol-4-yl)methoxy)-2,2-dimethyltetrahydrofuro $[2,3-d][1,3]$ dioxol-5-yl) - 3 - (4-fluorophenyl)-6-(4-nitrophenyl)-3,3a,5,6-tetrahydro- $2 H$-pyrazolo $[3,4-d]$ thiazole $(10 \mathrm{p})$. This was obtained by reacting compound 9c $(1 \mathrm{~g})$ and benzylhydrazine $(0.65 \mathrm{~g})$ as described in the typical procedure and isolated as a yellow solid, yield 71\%, m.p. $275-277{ }^{\circ} \mathrm{C}$; ${ }^{1} \mathrm{H}$ NMR $(300 \mathrm{MHz}$, DMSO-d $d_{6} \delta 8.35(\mathrm{~d}, J=8.7 \mathrm{~Hz}, 2 \mathrm{H}, \mathrm{Ar}-\mathrm{H}), 8.11(\mathrm{~s}, 1 \mathrm{H}$, Ar-H), 7.59 (d, J = 9.2 Hz, 2H, Ar-H), 7.46-7.40 (m, 6H, Ar-H and CH-S), 7.32 (d, $J=8.33 \mathrm{~Hz}, 2 \mathrm{H}, \mathrm{Ar}-\mathrm{H}), 7.08$ (d, $J=8.3 \mathrm{~Hz}, 2 \mathrm{H}, \mathrm{Ar}-\mathrm{H}), 6.96-6.90(\mathrm{~m}, 4 \mathrm{H}, \mathrm{Ar}-\mathrm{H}), 5.96(\mathrm{~s}$, 2H, $\left.\mathrm{CH}_{2} \mathrm{Ph}\right), 5.77$ (d, $\left.J=3.6 \mathrm{~Hz}, 1 \mathrm{H}, \mathrm{C}_{1} \mathrm{H}\right), 5.65$ (d, $J=2.2$ $\mathrm{Hz}, 1 \mathrm{H}, \mathrm{S}-\mathrm{CH}), 5.28$ (d, J=2.2 Hz, 1H, CH-N), 4.66 (t, $J=$ $\left.3.9 \mathrm{~Hz}, 1 \mathrm{H}, \mathrm{C}_{2} \mathrm{H}\right), 4.54\left(\mathrm{~s}, 2 \mathrm{H}, \mathrm{OCH}_{2}\right), 3.96-3.94(\mathrm{~m}, 1 \mathrm{H}$, $\left.\mathrm{C}_{4} \mathrm{H}\right), 3.26\left(\mathrm{dd}, J_{1}=9.1 \mathrm{~Hz}, J_{2}=4.2 \mathrm{~Hz}, 1 \mathrm{H}, \mathrm{C}_{3} \mathrm{H}\right), 1.53(\mathrm{~s}$, $\left.3 \mathrm{H}, \mathrm{CH}_{3}\right), 1.36\left(\mathrm{~m}, 3 \mathrm{H}, \mathrm{CH}_{3}\right) ;{ }^{13} \mathrm{C} \mathrm{NMR}(75 \mathrm{MHz}$, DMSO- $\left.d_{6}\right) \delta 171.6,151.6,143.2,137.4,133.6,132.3,131.2$, $129.3,125.3,128.4,127.9,124.8,122.9,119.2,111.2,103.8$, 81.2, 78.1, 74.1, 65.9, 58.1, 51.4, 26.1, 16.1; MS: $m / z\left(\mathrm{M}^{+}+\right.$ H) 773. Anal. Calcd for $\mathrm{C}_{39} \mathrm{H}_{35} \mathrm{ClFN}_{7} \mathrm{O}_{6} \mathrm{~S}$ : C, 59.73; H, 4.50; N, 12.56. Found: C, 59.66; H, 4.41; N, 12.36 .

5-((3aR,5S,6S,6aR)-6-((1-(4-Chlorophenyl)- $1 H-1,2,3-$ triazol-4-yl)methoxy)-2,2-dimethyltetrahydrofuro[2,3-d][1,3] dioxol-5-yl)-3-(4-fluorophenyl)-2-isopropyl-6-(4-nitrophenyl)-3,3a,5,6-tetrahydro- $2 \mathrm{H}$-pyrazolo[3,4-d] thiazole (10q). This was obtained by reacting compound 9c ( $1 \mathrm{~g})$ and isopropylhydrazine $(0.35 \mathrm{~g})$ as described in the typical procedure and isolated as a brown solid, yield 69\%, m.p. 221-223 ${ }^{\circ} \mathrm{C}$; ${ }^{1} \mathrm{H}$ NMR $(300 \mathrm{MHz}$, DMSO- $\left.d_{6}\right) \delta 8.32(\mathrm{~d}, J=8.7 \mathrm{~Hz}, 2 \mathrm{H}, \mathrm{Ar}-\mathrm{H}), 8.16(\mathrm{~s}, 1 \mathrm{H}$, Ar-H), 7.65 (d, J = 9.2 Hz, 2H, Ar-H), 7.52-7.48 (m, 5H, Ar-H and CH-S), 7.39 (d, $J=8.33 \mathrm{~Hz}, 2 \mathrm{H}, \mathrm{Ar}-\mathrm{H}), 7.09$ (d, $J=8.3 \mathrm{~Hz}, 2 \mathrm{H}, \mathrm{Ar}-\mathrm{H}), 5.76\left(\mathrm{~d}, J=3.6 \mathrm{~Hz}, 1 \mathrm{H}, \mathrm{C}_{1} \mathrm{H}\right), 5.62$ $(\mathrm{d}, J=2.2 \mathrm{~Hz}, 1 \mathrm{H}, \mathrm{S}-\mathrm{CH}), 5.25(\mathrm{~d}, J=2.2 \mathrm{~Hz}, 1 \mathrm{H}, \mathrm{CH}-\mathrm{N})$, $4.66\left(\mathrm{t}, J=3.9 \mathrm{~Hz}, 1 \mathrm{H}, \mathrm{C}_{2} \mathrm{H}\right), 4.54\left(\mathrm{~s}, 2 \mathrm{H}, \mathrm{OCH}_{2}\right), 3.96-3.94$ $\left(\mathrm{m}, 1 \mathrm{H}, \mathrm{C}_{4} \mathrm{H}\right), 3.26\left(\mathrm{dd}, J_{1}=9.1 \mathrm{~Hz}, J_{2}=4.2 \mathrm{~Hz}, 1 \mathrm{H}, \mathrm{C}_{3} \mathrm{H}\right)$, 2.80-2.78 (m, 1H, CH), $1.53\left(\mathrm{~s}, 3 \mathrm{H}, \mathrm{CH}_{3}\right), 1.36(\mathrm{~m}, 3 \mathrm{H}$, $\left.\mathrm{CH}_{3}\right), 0.96\left(\mathrm{~d}, J=6.3 \mathrm{~Hz}, 6 \mathrm{H}, 2 \times \mathrm{CH}_{3}\right) ;{ }^{13} \mathrm{C} \mathrm{NMR}(75 \mathrm{MHz}$, DMSO- $\left.d_{6}\right) \delta 171.6,151.6,143.2,137.4,133.6,132.3,131.2$, $129.3,128.4,127.9,124.8,122.9,119.2,111.2,103.8,81.2$, 78.1, 74.1, 65.9, 58.1, 51.4, 50.6, 26.1, 16.1; MS: $m / z\left(\mathrm{M}^{+}+\right.$ H) 736. Anal. Calcd for $\mathrm{C}_{35} \mathrm{H}_{35} \mathrm{ClFN}_{7} \mathrm{O}_{6} \mathrm{~S}$ : C, 57.10; H, 4.79; N, 13.32. Found: C, 56.98; H, 4.56; N, 12.99 .
5-((3aR,5S,6S,6aR)-6-((1-(4-Chlorophenyl)-1H-1,2,3triazol-4-yl)methoxy)-2,2-dimethyltetrahydrofuro[2,3-d][1,3] dioxol-5-yl)-3-(4-fluorophenyl)-2-methyl-6-(4-nitrophenyl)-3,3a,5,6-tetrahydro- $2 \mathrm{H}$-pyrazolo[3,4-d]thiazole (10r). This was obtained by reacting compound 9c ( $1 \mathrm{~g})$ and methylhydrazine $(0.25 \mathrm{~g})$ as described in the typical procedure and isolated as a brown solid, yield 67\%, m.p. $251-253{ }^{\circ} \mathrm{C} ;{ }^{1} \mathrm{H}$ NMR $(300 \mathrm{MHz}$, DMSO- $\left.d_{6}\right) \delta 8.29(\mathrm{~d}, J=8.7 \mathrm{~Hz}, 2 \mathrm{H}, \mathrm{Ar}-\mathrm{H}), 8.16(\mathrm{~s}, 1 \mathrm{H}$, Ar-H), 7.59 (d, $J=9.2 \mathrm{~Hz}, 2 \mathrm{H}, \mathrm{Ar}-\mathrm{H}), 7.48-7.44$ (m, 5H, $\mathrm{Ar}-\mathrm{H}$ and CH-S), 7.39 (d, $J=8.33 \mathrm{~Hz}, 2 \mathrm{H}, \mathrm{Ar}-\mathrm{H}), 7.09$ (d, $J=8.3 \mathrm{~Hz}, 2 \mathrm{H}, \mathrm{Ar}-\mathrm{H}), 5.76\left(\mathrm{~d}, J=3.6 \mathrm{~Hz}, 1 \mathrm{H}, \mathrm{C}_{1} \mathrm{H}\right), 5.62$ $(\mathrm{d}, J=2.2 \mathrm{~Hz}, 1 \mathrm{H}, \mathrm{S}-\mathrm{CH}), 5.25(\mathrm{~d}, J=2.2 \mathrm{~Hz}, 1 \mathrm{H}, \mathrm{CH}-\mathrm{N})$, $4.66\left(\mathrm{t}, J=3.9 \mathrm{~Hz}, 1 \mathrm{H}, \mathrm{C}_{2} \mathrm{H}\right), 4.54\left(\mathrm{~s}, 2 \mathrm{H}, \mathrm{OCH}_{2}\right), 3.96-3.94$ $\left(\mathrm{m}, 1 \mathrm{H}, \mathrm{C}_{4} \mathrm{H}\right), 3.71\left(\mathrm{~s}, 3 \mathrm{H}, \mathrm{N}-\mathrm{CH}_{3}\right), 3.26\left(\mathrm{dd}, J_{1}=9.1 \mathrm{~Hz}, J_{2}\right.$ $\left.=4.2 \mathrm{~Hz}, 1 \mathrm{H}, \mathrm{C}_{3} \mathrm{H}\right), 1.53\left(\mathrm{~s}, 3 \mathrm{H}, \mathrm{CH}_{3}\right), 1.36\left(\mathrm{~m}, 3 \mathrm{H}, \mathrm{CH}_{3}\right)$; ${ }^{13} \mathrm{C}$ NMR $\left(75 \mathrm{MHz}, \mathrm{DMSO}-d_{6}\right) \delta 171.6,151.6,143.2,137.4$, 133.6, 132.3, 131.2, 129.3, 128.4, 127.9, 124.8, 122.9, 119.2, 111.2, 103.8, 81.2, 78.1, 74.1, 65.9, 58.1, 51.4, 39.3, 16.1; MS: $m / z\left(\mathrm{M}^{+}+\mathrm{H}\right)$ 684. Anal. Calcd for $\mathrm{C}_{33} \mathrm{H}_{31} \mathrm{ClFN}_{7} \mathrm{O}_{6} \mathrm{~S}: \mathrm{C}$, 55.97; H, 4.41; N, 13.85. Found: C, 55.82; H, 4.26: N, 13.72.

\section{Conclusion}

In conclusion, a series of a new class of hybrid heterocycles 10a-r have been synthesized and evaluated for their nematicidal activity, most of the compounds showed appreciable nematicidal activity. The antifungal activities of these compounds were evaluated against various fungi. Many of the synthesized compounds showed good activity against the tested fungi and therefore have emerged as potential molecules for further development.

\section{Acknowledgements}

The authors are thankful to CSIR-New Delhi for the financial support (Project funding No: 02 (247)15/EMRII), Director, CSIR- IICT, Hyderabad, India, for NMR and MS spectral analysis and Principal, Vaagdevi Degree \& PG College Hanamkonda for his constant encouragement.

\section{References}

1. (a) K. D. Hani, D. A. Leigh, Chem. Soc.Rev. 2010, 39, 12401251. DOI:10.1039/B901974J

(b) C. O. Kappe, E. Van der Eycken, Chem.Soc. Rev. 2010, 39, 1280-1290 DOI:10.1039/B901973C

(c) A. H. El-Sagheer, T. Brown, Chem. Soc. Rev. 2010, 39, 1388-1405 DOI:10.1039/b901971p

(d) A. Qin, J. W. Y. Lam, B. Z. Tang, Chem. Soc. Rev. 2010, 2522-2544. DOI:10.1039/b909064a

(e) M. Meldal, C. W. Tornoe, Chem. Rev. 2008, 108, 29523015. DOI:10.1021/cr0783479 
(f) H. Nandivada, X. Jiang, J. Lahann, Adv. Mater. 2007, 19, 2197-2208. DOI:10.1002/adma.200602739

(g) Y. L. Angell, K. Burgess, Chem. Soc. Rev. 2007, 36, 1674 1689. DOI:10.1039/b701444a

(h) D. Fournier, R. Hoogenboom, U. S. Schubert, Chem. Soc. Rev. 2007, 36, 1369-1380. DOI:10.1039/b700809k

(i) J. E. Moses, A. D. Moorhouse, Chem. Soc. Rev. 2007, 36, 1249-1262. DOI:10.1039/B613014N

(j) J. F. Lutz, Angew. Chem. Int. Ed. 2007, 46, 1018-1125.

DOI:10.1002/anie.200604050

(k) A. Dondoni, Chem. - Asian J. 2007, 2, 700-708.

DOI:10.1002/asia.200700015

(1) H. C. Kolb, K. B. Sharpless, Drug Discovery Today, 2003, 8, 1128-1137. DOI:10.1016/S1359-6446(03)02933-7

2. (a) A. Brick, J. Muldoon, Y.-C. Lin, J. H. Elder, D. S. Goodsell, A. J. Olson, V. V. Fokin, K. B. Sharpless, C.-H. Wong, ChemBioChem. 2003, 4, 1246-1248. DOI:10.1002/cbic.200300724 (b) M. J. Soltis, H. J. Yeh, K. A. Cole, N. Whittaker, R. P. Wersto, E. C. Kohn, Drug Metab. Dispos.1996, 24, 799-806.

3. (a) W.-Q. Fan, A. R. Katritzky, 1,2,3-Triazoles, In Comprehensive Heterocyclic Chemistry II. Edited by A. R. Katritzky, C. W. Rees, V. Scriven, Elsevier, Oxford. 1996, 4, 1-126, pp. 905-1006.

(b) M. Whiting, J. Muldoon, Y.-C. Lin, S. M. Silverman, W. Lindstrom, A. J. Olson, H. C. Kolb, M. G. Finn, K. B. Sharpless, J. H. Elder, V. V. Fokin, Angew. Chem. Int. Ed., 2006, 45, 1435-1439. DOI:10.1002/anie.200502161

(c) Y. Bourne, H. C. Kolb, Z. Radić, K. B. Sharpless, P.Taylor, P. Marchot, Proc. Natl. Acad. Sci. U.S.A. 2004, 101, 1449-1454. DOI:10.1073/pnas.0308206100

(d) W. G. Lewis, G. Green, F. Z. Grynszpan, Z. Radić, P. R. Carlier, P. Taylor, M. G. Finn, K. B. Sharpless, Angew. Chem., Int. Ed. 2002, 41, 1053-1057.

DOI:10.1002/1521-3773(20020315)41:6<1053::AID-ANIE 1053>3.0.CO;2-4

4. R. Huisgen, A. Padwa, 1,3-Dipolar Cycloaddition Chemistry, Ed. Wiley, New York, 1984, 1, 1-176.

5. (a) N. A. Al-Maoudi, A. Y. Al-Soud, Tetrahedron Lett. 2002, 43, 4021-4022. DOI:10.1016/S0040-4039(02)00733-5

(b) B. H. M. Kuijpers, S. Groothuys, A. B. R. Keereweer, P. J. L. M. Quaedflieg, R. H. Blaauw, F. L.van Delft, F. P. J. T. Rutjes, Org. Lett. 2004, 6, 3123-3126. DOI:10.1021/ol048841o

(c) C. Srinivas, X. Fang, Q. Wang, Tetrahedron Lett. 2005, 46, 2331-2334. DOI:10.1016/j.tetlet.2005.01.175

(d) S. Hotha, R. I. Anegundi, A. A. Natu, Tetrahedron Lett. 2005, 46, 4585-4588. DOI:10.1016/j.tetlet.2005.05.012

(e) S. Hotha, S. Kashyap, J. Org. Chem. 2006, 71, 364-367. DOI:10.1021/jo051731q

6. H. K. Urman, O. Bulay, B. Clayson, P. Shudik, Cancer Lett. 1975, 1, 69-74. DOI:10.1016/S0304-3835(75)95362-8

7. A. A. Field, A. A. Tyrell, G. P. Lampson, M. R. Hilleman, Proc. Natl. Acad. Sci. U.S.A, 1967, 58, 1004-1010.

DOI:10.1073/pnas.58.3.1004

8. M. G. Vigorita, R. Ottana, F. Monoforte, R. Maccari, A. Trovato, M. T. Monoforte, M. F. Taviano, Bioorg. Med. Chem. Lett. 2001, 11, 2791-2794.
DOI:10.1016/S0960-894X(01)00476-0

9. M. S. Chande, V. J. Suryanarayana, J. Chem. Res, 2005, 345347. DOI:10.3184/0308234054506749

10. C. V. Kavitha, S. Basappa, N. Swamy, K. Manteling, S. Doreswamy, M. A. Sridhar, S. Prasad, K. S. Rangappa, Bioorg. Med. Chem. Lett. 2006, 14, 2290-2299.

DOI:10.1016/j.bmc.2005.11.017

11. B. A. Sobin, J. Am. Chem. Soc, 1952, 74, 2947-2948.

DOI:10.1021/ja01131a526

12. Y. Tanabe, Y. Kumuro, N. Imanishi, S. Morooka, M. Enomoto, A. Kojima, Y. Sanemistu, M. Mizutani, Tetrahedron Lett. 1991, 32, 379-382.

DOI:10.1016/S0040-4039(00)92633-9

13. T. Previtera, M. G. Vigorita, M. Bisila, F. Orshini, F. Benetolla, G. Bombieri, Eur. J. Med. Chem. 1994, 29, 317-324.

DOI:10.1016/0223-5234(94)90102-3

14. R. Ottana, E. Mazzon, L. Dugo, F. Moforte, R. Maccari, L. Sautebin, G. De Luca, M. G. Vigorita, S. Alcaro, F. Ortuso, Eur. J. Pharmacol. 2002, 448, 71-80.

DOI:10.1016/S0014-2999(02)01888-5

15. T. Kato, T. Ozaki, K. Tamura, J. Med. Chem. 1999, 42, 3134 3146. DOI:10.1021/jm9900927

16. Y. Tanabe, G. Suzukamo, Y. Komuro, N. Imanishi, S. Morroka, M. Enomoto, A. Kojima, Y. Sanimistu, M. Mizutani, Tetrahedron Lett. 1991, 32, 379-382. DOI:10.1016/S0040-4039(00)92633-9

17. T. Kato, T. Ozaki, K.Tamura, Tetrahedron Assymm. 1999, 10, 3963-3968. DOI:10.1016/S0957-4166(99)00441-3

18. Y. Adachi, Y. Suzuki, N. Homma, M. Fuzukawa, K. Tamura, I. Nishie, O. Kuromaru, Eur. J. Pharmacol. 1999, 367, 267-273. DOI:10.1016/S0014-2999(98)00938-8

19. C. S. Reddy, A. Srinivas, A. Nagaraj, J. Heterocycl. Chem. 2008, 45, 999-1003. DOI:10.1002/jhet.5570450409

20. K. Y. Lee, J. M. Kim, J. N. Kim, Tetrahedron Lett. 2003, 44, 6737-6740. DOI:10.1016/S0040-4039(03)01648-4

21. P. Erhan, A. Mutlu, U. Tayfun, E. Dilek, Eur. J. Med. Chem. 2001, 36, 539-543. DOI:10.1016/S0223-5234(01)01243-0

22. R. A. Nugen, M. Meghan, J. Med. Chem. 1993, 36, 134-139. DOI:10.1021/jm00053a017

23. H. Kawazura, Y. Takahashi, Y. Shiga, F. Shimada, N. Ohto, A. Tamura, Jpn. J. Pharmacol. 1997, 73, 317-324. DOI:10.1254/jjp.73.317

24. M. J. Genin, C. Bilers, B. J. Kieser, S. M. Poppe, S. M. Swaney, W. G. Tarpley, Y. Yagi, D. L. Romero, J. Med. Chem. 2000, 43, 1034-1040. DOI:10.1021/jm990383f

25. A. G. Habeb, P. N. P. Rao, E. E. Knaus, J. Med. Chem. 2001, 44, 3039-3042. DOI:10.1021/jm010153c

26. H. Hashimoto, K. Imamura, J. I. Haruta, K. Wakitani, J. Med. Chem. 2002, 45, 1511-1517. DOI:10.1021/jm010484p

27. M. Sakya, B. Rast, Tetrahedron Lett. 2003, 44, 7629-7632. DOI:10.1016/j.tetlet.2003.08.054

28. L. David, D. G. B. Selwood, J. Med. Chem. 2001, 44, 78-93. DOI:10.1021/jm001034k

29. U. L. B. Jayasinghe, B. M. M. Kumarihamy, A. G. D. Bandara, E. A. Vasquez, W. Kraus, Nat. Prod. Res. 2003, 17, 259-262. DOI:10.1080/1057563021000060121 
30. S. Min, L. Xiaoming, J. Shuishui, L. Lingjie, W. Jiali, W. Shiqiang, Applied Soil Ecology. 2016, 102, 53-60. DOI:10.1016/j.apsoil.2016.02.011

31. J. W. Noling, J. O. Becker, J. Nematol. 1994, 26, 573-586.

32. C. In Ho, K. Jun Heon, S. Sang-Chul, P. Il-Kown, Rus. J. Nematology 2007, 15, 35-40.

33. K. Shigefumi, W. Kojiro, M. Katsura, Agr. Bio. Chem. 1976, 40, 2085-2089. DOI:10.1080/00021369.1976.10862338

34. J. H. Uhlenbroek, J. D. Bijloo, Recl. Trav. Chim. 1958, 77, 1004-1008. DOI:10.1002/recl.19580771103

35. (a) A. Srinivas, M. Sunitha, B. Ravinder, S. Anusha, T. Rajasri, P. Swapna, D. Sushmitha, D. Swaroopa, G. Nikitha, C. Govind Rao, Acta Chim. Slov. 2017, 64, 319-331.

DOI:10.17344/acsi.2016.3153

(b) A. Srinivas, M. Santhosh, M. Sunitha, P. Karthik, K. Srinivas, K. Vasumathi Reddy, Acta Chim. Slov. 2016, 63, 827-836. DOI:10.17344/acsi.2015.2124

36. A. Srinivas, M. Sunitha, C. Govind Rao, Acta Chim. Slov. 2016, 63, 344-350. DOI:10.17344/acsi.2015.2124

37. A. Srinivas, Acta Chim. Soc. 2016, 63, 173-179.

DOI:10.17344/acsi.2015.2124

38. A. Srinivas, M. Sunitha, Indian J. Chem., Sect. B 2016, 55B, 102-109.

39. A. Srinivas, M. Sunitha, Indian J. Chem., Sect. B 2016, 55B, 231-239.
40. A. Srinivas, M. Sunitha, C. Govind Rao, Indian J. Chem., Sect. B 2016, 55B, 1239-1242.

41. A. Srinivas, A. Nagaraj, C. S. Reddy, Eur. J. Med. Chem. 2010, 45, 2353-2358. DOI:10.1016/j.ejmech.2010.02.014

42. C. S. Reddy, A. Srinivas, M. Sunitha, A. Nagaraj, J. Heterocycl. Chem, 2010, 47, 1303-1309. DOI:10.1002/jhet.474

43. C. S. Reddy, A. Nagaraj, A. Srinivas, G. P. Reddy, Indian J. Chem., Scet. B, 2010, 49B, 617-622.

44. C. S. Reddy, A. Srinivas, A. Nagaraj, J. Heterocycl. Chem. 2009, 46, 497-502. DOI:10.1002/jhet.100

45. C. S. Reddy, A. Nagaraj, A. Srinivas, G. P. Reddy, Indian J. Chem., Sect. B 2009, 48B, 248-254.

46. C. S. Reddy, A. Srinivas, A. Nagaraj, J. Heterocyclic. Chem. 2008, 45, 1121-1125.

47. C. S. Reddy, G. P. Reddy, A. Nagaraj, A. Srinivas, Org. Commun. 2008, 1, 84-94.

48. A. Srinivas, A. Nagaraj, C. S. Reddy, Indian J. Chem., Sect. B 2008, 47B, 787-791.

49. P. A. Levene, G. M. Meyer, J. Biol. Chem, 1931, 92, 257.

50. National Committee for clinical laboratory standards (NCCLS). Nat. Comm. Lab. Stands. Villanova, 1982, p. 242.

51. Z. K. Khan, Proc. Int. Workshop UNIDO-CDRI, 1997, 210 (1997).

52. C. W. McBeth, G. B. Bergerson, Phytopathology 1953, 43, 264.

\section{Povzetek}

$\mathrm{Z}$ reakcijo med halkonskimi derivati 2-((3aR,5S,6S,6aR)-6-((1-(4-klorofenil)-1H-1,2,3-triazol-4-il)metoksi)-2,2-dimetiltetrahidrofuro[2,3-d][1,3]dioksol-5-il)-3-feniltiazolidin-4-onov 9 in aril/alkil hidrazini smo pripravili novo serijo 5-((3aR,5S,6S,6aR)-6-((1-(4-klorofenil)-1H-1,2,3-triazol-4-il)metoksi)-2,2-dimetiltetrahidrofuro[2,3- $d][1,3]$ dioksol5-il)-3-(4-fluorofenil)-2,6-difenil-3,3a,5,6-tetrahidro-2H-pirazolo[3,4-d]tiazolov 10a-r. Strukture novih spojin smo določili na osnovi IR, NMR, MS in elementne analize. Za spojine 10a-r smo tudi določili učinkovanje proti ploskim črvom (nematodam) Dietylenchus myceliophagus in Caenorhabditis elegans z in vitro metodo v vodnih raztopinah. Ugotovili smo, da spojine, ki vsebujejo $N$-benzilpirazolni (10d, 10j, 10p) ali $N$ - metilpirazolni fragment (10f, 10i, 10r), izkazujejo občutno nematocidno aktivnost proti obema testiranima živalskima vrstama $\mathrm{z}_{50} \mathrm{LD}_{50}$ 160 $210 \mathrm{ppm}$, kar je skoraj enako aktivnosti standarda oksamila. Za spojine 10a-r smo tudi raziskali delovanje proti glivam (izmerili smo MZI, MIC in MFC vrednosti) in sicer: Candida albicans (ATCC 102331), Aspergillus fumigates (HIC 6094), Trichophyton rubrum (IFO 9185) in Trichophyton mentagrophytes (IFO 40996). Večina novih spojin je izkazala opazno delovanje proti testiranim glivam, kar daje možnosti nadaljnjega razvoja predstavljenih spojin 